Illinois State University

ISU ReD: Research and eData

Theses and Dissertations

$10-10-2017$

\title{
Communicating consent: An investigation of predictors of college student sexual consent communication
}

Erin L. Link

Illinois State University, ellink@ilstu.edu

Follow this and additional works at: https://ir.library.illinoisstate.edu/etd

Part of the Communication Commons, and the Public Health Education and Promotion Commons

\section{Recommended Citation}

Link, Erin L., "Communicating consent: An investigation of predictors of college student sexual consent communication" (2017). Theses and Dissertations. 819.

https://ir.library.illinoisstate.edu/etd/819

This Thesis is brought to you for free and open access by ISU ReD: Research and eData. It has been accepted for inclusion in Theses and Dissertations by an authorized administrator of ISU ReD: Research and eData. For more information, please contact ISUReD@ilstu.edu. 


\title{
COMMUNICATING CONSENT: AN INVESTIGATION OF PREDICTORS OF COLLEGE STUDENT SEXUAL CONSENT COMMUNICATION
}

\author{
Erin L. Link
}

\section{Pages}

Often, sexual consent is only discussed and explored in the context of sexual assault. While consent is often the deciding factor in determining if a crime occurred, consent is also a vital part of an equally enjoyable sexual encounter. The present study examines a variety of variables effects on consent attitudes, norms, and communication behaviors to attempt to identify predictors of college student consent communication. Findings and recommendations are discussed that highlight the necessity of viewing consent as a communicative act.

KEYWORDS: empathy, perceived behavioral control, sexual consent, sex communication 


\author{
ERIN L. LINK
}

A Thesis Submitted in Partial Fulfillment of the Requirements for the Degree of

MASTER OF SCIENCE

School of Communication

\title{
ILLINOIS STATE UNIVERSITY
}


(C) 2017 Erin L. Link 


\author{
ERIN L. LINK
}

COMMITTEE MEMBERS:

Kevin R. Meyer, Chair

Lance R. Lippert

Aimee E. Miller-Ott 


\section{ACKNOWLEDGMENTS}

First off, I must thank Dr. Kevin Meyer for his guidance, knowledge, and unwavering patience while serving as my committee chair. From repeatedly explaining statistical tests to parenting tips, I would have not gotten through the past few years without your assistance. Your logical and methodical approach was the perfect match for what began as a fragmented, disorganized jumble of ideas. Thank you for always pushing me and believing in my project.

Between my undergraduate and graduate degrees, I have spent a total of six years going in and out of Fell Hall. While I am appreciative for all of the professors I experienced during my time at the School of Communication, I am particularly grateful to my thesis committee members Drs. Lance Lippert and Aimee Miller-Ott. Thank you not only for serving on my committee, but also for providing guidance and support throughout my time in graduate school. The knowledge you have shared about communication and beyond has been invaluable.

They say it takes a village to raise a child, and this is also true for completing a thesis. I could not have done this without the amazing support system of my family, friends, and fantastic co-workers. From assisting with childcare to supplying endless amounts of caffeine, no act of kindness and patience has gone unnoticed. Your assistance over the past four years has been instrumental to me completing this research project and degree.

Lastly, thank you my husband Jason and daughter Elliotte. Jason, thank you for becoming the domestic goddess you never knew you wanted to be and not letting me quit. Ellie, I hope you grow up in a world where respecting yourself and others in all situations is the norm. This project is my small contribution to help make that aspiration a reality. Thank you both for always supporting me, even when it meant time away from our family. You two are my world.

E. L. L. 


\section{CONTENTS}

\section{Page}

ACKNOWLEDGMENTS

CONTENTS

ii

TABLES

vi

FIGURES

vii

CHAPTER I: REVIEW OF LITERATURE

Defining Sexual Consent

Consent as a Communicative Act

Consent's Relationship with Sexual Assault

Applying the Social-ecological Model

Consent at the Individual Level

Perceived behavioral control

Empathy and consent

Consent at the Interpersonal Level

Communicating consent

Sex scripts

Metacommunication

Consent at the Community Level

Unique challenges of college campuses

Poor sexual preparation of college students

Consent at the Societal Level

Rape culture and rape myths 
$\begin{array}{ll}\text { CHAPTER II: METHOD } & 30\end{array}$

$\begin{array}{ll}\text { Participants } & 30\end{array}$

$\begin{array}{ll}\text { Procedures } & 31\end{array}$

$\begin{array}{ll}\text { Measures } & 32\end{array}$

$\begin{array}{ll}\text { Empathy } & 32\end{array}$

$\begin{array}{ll}\text { Perceived Behavioral Control } & 33\end{array}$

$\begin{array}{ll}\text { Attitudes toward Establishing Consent } & 34\end{array}$

Approaches to Establishing Consent 34

$\begin{array}{ll}\text { Consent Norms } & 35\end{array}$

$\begin{array}{ll}\text { Ongoing Negotiation } & 36\end{array}$

$\begin{array}{ll}\text { Metacommunication } & 39\end{array}$

$\begin{array}{ll}\text { Data Analysis } & 41\end{array}$

$\begin{array}{ll}\text { CHAPTER III: RESULTS } & 44\end{array}$

Consent at the Individual Level $\quad 44$

$\begin{array}{ll}\text { Perceived Behavioral Control } & 44\end{array}$

$\begin{array}{ll}\text { Empathy } & 45\end{array}$

Consent at the Interpersonal Level 46

Nontraditional Gender Identities and Sexual Orientations 46

$\begin{array}{ll}\text { Metacommunication } & 49\end{array}$

$\begin{array}{lr}\text { Consent at the Community Level } & 50\end{array}$

$\begin{array}{lr}\text { Prior Consent Education } & 50\end{array}$

$\begin{array}{ll}\text { Consent at the Societal Level } & 51\end{array}$ 
Sources of Influence $\quad 51$

$\begin{array}{ll}\text { Additional Findings } & 52\end{array}$

$\begin{array}{ll}\text { CHAPTER IV: DISCUSSION } & 57\end{array}$

$\begin{array}{ll}\text { Summary of Findings } & 58\end{array}$

$\begin{array}{ll}\text { Consent at the Individual Level } & 58\end{array}$

$\begin{array}{lr}\text { Perceived behavioral control } & 58\end{array}$

$\begin{array}{ll}\text { Empathy } & 59\end{array}$

$\begin{array}{ll}\text { College student preparation } & 61\end{array}$

$\begin{array}{ll}\text { Year in school } & 62\end{array}$

Interpersonal Aspects of Consent $\quad 63$

$\begin{array}{lr}\text { Sexual and gender minorities } & 63\end{array}$

$\begin{array}{ll}\text { Consent as an ongoing process } & 64\end{array}$

$\begin{array}{ll}\text { Community Impacts on Consent } & 65\end{array}$

$\begin{array}{ll}\text { Prior consent education } & 65\end{array}$

$\begin{array}{ll}\text { Alcohol and college } & 67\end{array}$

$\begin{array}{ll}\text { Societal Influences on Consent } & 69\end{array}$

$\begin{array}{ll}\text { Implications } & 71\end{array}$

$\begin{array}{ll}\text { The Role of Communication } & 71\end{array}$

$\begin{array}{ll}\text { Interpersonal Skill Building } & 73\end{array}$

$\begin{array}{ll}\text { Addressing Community and Societal Influences } & 76\end{array}$

$\begin{array}{ll}\text { Study Limitations } & 79\end{array}$

Opportunities for Future Research $\quad 81$

$\begin{array}{ll}\text { Conclusion } & 82\end{array}$ 


\section{TABLES}

Table

Page

1. Factor Loadings for Consent Norms Scale

2. Factor Loadings for Ongoing Negotiation Scale

3. Factor Loadings for Metacommunication Scale

4. Beta Weights for Perceived Behavioral Control Model

5. Descriptive Statistics for Sexual Identity

6. Descriptive Statistics for Gender Identity

7. Beta Weights for Consent as an Ongoing Process Model

8. Descriptive Statistics for Prior Consent Education

9. Descriptive Statistics for Sources of Influence

10. Correlations among Scales

11. Descriptive Statistics for Scales by Year in School 


\section{FIGURES}

Figure $\quad$ Page

1. Social-ecological Model $\quad 8$ 


\section{CHAPTER I: REVIEW OF LITERATURE}

Sexual consent is a widely discussed, but largely misunderstood concept with definitions and understandings varying greatly between scholars, legal sources, and the general public. One reason that sexual consent has garnered increasing attention is because of its relationship with sexual assault. Recent estimates indicate that nearly one in five women experience sexual assault while in college (White House Council on Women and Girls, 2014; Gray, 2014). However, consent's inextricable relationship with sexual assault is problematic. Sexual consent is often thought about, and largely defined, in the context of non-consent (Cowling \& Reynolds, 2004). Consent is an important concept beyond situations of assault, coercion, and rape. Successful negotiation of consent is a crucial component to ensuring both partners are interested and willing to participate, thus securing a pleasurable sexual experience enjoyed equally by both partners (Flyntz, 2016).

Successful consent communication is also vital to ethical, sexual decision making and overall happiness (Cowling \& Reynolds, 2004). Even though consent is a communicative phenomenon, the majority of existing research comes from disciplines such as psychology and sociology-and is largely addressed in the context of rape and coercion. Many young adults believe that sexual miscommunication occurs frequently and sometimes causes sexual assaults (Derning, Krassen Covan, Swan, \& Billings, 2013; Johnson \& Hoover, 2015; Vandiver \& Rager Dupalo, 2012). The most widely accepted rape myth among college students excuses the perpetrator because he/she did not mean to commit the rape (Vandiver \& Rager Dupalo, 2012). However, attributing sexual assault to a misunderstanding perpetuates a culture supportive of violence (White House Council on Women and Girls, 2014). Even survivors of rape sometimes 
attribute the assault to miscommunication, reporting that they felt led on by their attacker (Lim \& Roloff, 1999).

Attributing sexual assault to miscommunication is highly problematic. Sexual assault crimes are committed by perpetrators who do not intend to obtain consent or heed consent signals from their victims (Lisak \& Miller, 2002). For this reason, this project does not focus on sexual assault. Nonetheless, sexual assault and consent are intimately linked for many reasons including the often unclear definition of sexual consent and the frequent indirect, nonverbal communication of consent (Jozkowski \& Peterson, 2014). Even though previous research exists, researchers and educators do not fully understand how college students conceptualize or define consent (Beres, 2007; Donat \& White, 2000). In addition, legal definitions of consent as well as definitions discussed in peer-reviewed literature lack consistency and likely do not reflect how college students conceptualize consent (Beres, 2014).

This study aims to advance existing research on college students' consent behaviors and attitudes from a communicative lens. By applying the social-ecological model, this study examines factors that affect consent at the individual, interpersonal, community, and societal levels. At the individual level, this study investigates the effect of perceived behavioral control and empathy on consent. The interpersonal level assesses sex scripts and metacommunication, or communication partners have about the way they communicate. Community aspects of college campuses and societal elements that influence consent are also discussed. In addition, the present study examines consent behaviors and norms for sexual minorities, which is an area of research that is largely unexplored. Knowing more about how college students communicate consent, especially those that identify as sexual minorities, will enhance communication scholars' and 
college health professionals' ability to create better informed sexual consent education initiatives.

\section{Defining Sexual Consent}

Sexual consent is a complicated and contentious concept for both health professionals and the public at large. Definitions vary widely among legal, scholarly, and popular press sources (Beres, 2014). The term consent is frequently employed without providing explanation, mistakenly assuming a mutual understanding of the concept (Beres, 2007, 2014). Moreover, consent is often defined in terms of non-consent, which adds to the uncertainty surrounding a common definition (Cowling \& Reynolds, 2004). Furthermore, most scholarly and legal explanations do not take into consideration how individuals conceptualize, define, and communicate consent.

At its most basic level, sexual consent is some form of an agreement to engage in sexual activity. Most scholarly and legal sources agree that individuals must grant consent freely, without any coercion or force (Hickman \& Muehlenhard, 1999; Lim \& Roloff, 1999; Muehlenhard, Humphreys, Jozkowski, \& Peterson, 2016). One element that adds to the complexity of consent is that definitions vary widely by jurisdiction and organization (Beres, 2014; Muehlenhard et al., 2016). Part of the reason that definitions vary widely is the fact that consent is typically defined in terms of non-consent with a focus on rape, forced sex, and abuse (Cowling \& Reynolds, 2004; Reynolds, 2004).

There are two main components to the legal definition of consent. First, legal definitions outline who is competent to give consent as determined by the individual's age and mental capacity (Beres, 2014). The second element of consent's legal definition includes how consent is communicated. Again, legal definitions vary widely. Some definitions consider consent to 
include any agreement to participate in sex, even if the individual is coerced (McGregor, 1996; West, 1996). In contrast, some jurisdictions consider consent to only be established in the absence of coercion or force (Beres, 2014). Certain jurisdictions also detail the types of communication that can indicate consent. Typically, college students have at least two legal authorities defining consent for them: their institution's code of conduct and the laws of the region where they live.

Focusing solely on legal definitions of consent is problematic. Varying legal definitions do not take into account personal and social identities as well as cultural differences, all of which are important factors that affect consent decisions (Reynolds, 2004). This is particularly concerning for college-aged individuals because current definitions do not reflect how young adults conceptualize, define, and communicate consent (Cowling \& Reynolds, 2004). Understanding how college students approach consent decisions is vital because trying to impose patterns of behaviors outside of their norms may be perceived as forced and artificial.

Additional factors to take into consideration when defining consent are the relationship between the individuals involved the sexual encounter and the type of sexual activity performed. If individuals are in a romantic relationship, the longer they have been in the relationship the less likely they are to obtain consent (Humphreys, 2007; Spence, Losoff, \& Robbins, 1991;

Struckman-Johnson, 1988). In addition, the more individuals perceive a sexual act as intimate, the more likely they are to obtain overt consent (Humphreys, 2007). Individuals report that penetrative sexual acts, such as oral, vaginal, or anal sex, require more explicit consent than other behaviors that are perceived as less intimate, such as kissing and touching (Humphreys, 2004). 


\section{Consent as a Communicative Act}

Scholarly, legal, and general conversations about consent largely ignore the communicative components of consent situations. Most existing scholarly research on consent comes from the disciplines of psychology and sociology, and approach consent within the context of coercion and rape (Cowling \& Reynolds, 2004). And, given the lack of consensus among legal sources and scholars as to the definition of consent, it is no surprise that consent communication is largely a matter of individual interpretation and the ability to attribute consent fluctuates (Hickman \& Muehlenhard, 1999; Jozkowski et al., 2014; Lim \& Roloff, 1999; Lindgren, Parkhill, George, \& Henderson, 2008). Since the majority of sexual interactions are not assaults, many researchers assume that individuals are able to successfully communicate sexual intent (Jozkowski et al., 2014). For many reasons this is faulty logic; perhaps, most importantly, because sexual assault is a grossly underreported crime; many victims do not even realize that they have experienced a criminal act (White House Council on Women and Girls, 2014). However, the threshold for what constitutes a consensual sexual encounter should not simply be to determine if a crime has or has not taken place. Consent's importance extends far beyond criminality. Securing blatant consent ensure a pleasurable sexual encounter equally enjoyed by both partners and contributes to the overall sexual enjoyment and happiness of individuals involved (Cowling \& Reynolds, 2004; Flyntz, 2016).

What is largely missing from scholarly and legal sources is an explicit focus on the communicative elements of consent as well as the widespread acceptance that consent communication is ongoing throughout the entire sexual encounter (Reynolds, 2004). For the purposes of this study, sexual consent is defined as a series of communicative acts that freely convey mutual agreement to engage in sexual acts through verbal and nonverbal signals. Consent 
decisions must include a communicative signifier. Consent decisions - sexual in nature or otherwise - are procedural and communicative signifiers imply change in personal desires to proceed (Reynolds, 2004). Personal attributes and cognitive processes are important elements that influence consent communication and the processing of consent communication. However, in order for consent to take place, individuals must move beyond cognition and communicate in clear, unambiguous manner with their partner. While actions can be used to help support words, verbal communication is necessary for securing affirmative consent.

\section{Consent's Relationship with Sexual Assault}

Even though the present study does not examine sexual assault, it is important to understand consent's relationship with sexual assault in order to fully explore all factors that affect consent. Consent is inextricably tied to sexual assault because sexual-based crimes are often defined in terms of consent, or lack thereof (Jozkowski \& Peterson, 2013; Muehlenhard et al., 2016). Additionally, lack of consent is often the central feature of legal research and definitions of rape and sexual assault (Jozkowski et al., 2014). Sexual assault or rape occurs when partners either do not obtain consent or disregard refusals to engage in sexual activity (Hust et al., 2014). Communication is often regarded as the key difference between sexual victimization and a consensual sexual experience (Jozkowski \& Peterson, 2014). For this reason, sexual assault prevention efforts often hinge on clarifying the definition of and promoting the obtainment of consent as a mechanism to reduce sexual assault.

However, the importance of consent extends beyond ensuring that individuals obtain proper permission for sexual activity. Consent ensures both partners are interested in and willing to participate, guaranteeing a pleasurable sexual experience enjoyed equally by both partners (Cowling \& Reynolds, 2004; Flyntz, 2016; Reynolds, 2004). Improved consent communication 
may also reduce the amounts of miscommunication and misinformation surrounding consent. Lastly, heightening knowledge about consent at a societal level can also reduce rape-supportive myths that are widely accepted, especially in the college population (Vandiver \& Rager Dupalo, 2012).

Even though it is important to give context to consent in regards to sexual assault, this study will not investigate any aspects of sexual assault since assaults lack consent communication involved. Aggressors who repeat their crimes commit the majority of college sexual assaults, with some perpetrators admitting to committing as many as six sexual assaults (White House Council on Women and Girls, 2014). Even when perpetrators engage in consent communication, they do not intend to acknowledge and/or respect their partner's consent signals (Lisak \& Miller, 2002). While many sexual assault prevention programs focus on consent communication as a means of preventing crimes, the only person who can prevent a sexual assault is the perpetrator. Instead, the present study concentrates on consent as an important and necessary component to mutually enjoyable and ethical sexual encounters.

\section{Applying the Social-ecological Model}

One way to explain all of the complex elements that affect consent communication is to apply the social-ecological model. According to the Centers for Disease Control and Prevention (CDC) (2015), the social-ecological model is a theoretical approach to public health that takes into account the complex interplay among individual, interpersonal, community, and societal factors that affect health. The overlapping rings of the model illustrate how elements in each level influences factors at another level (see Figure 1). Some iterations of the social-ecological model include additional layers, such as organization and policy. Ecological models are 
important in social science research because the approach views behavior as influenced by, as well as affecting, the social environment (McLeroy, Bibeau, Steckler, \& Glans, 1988).

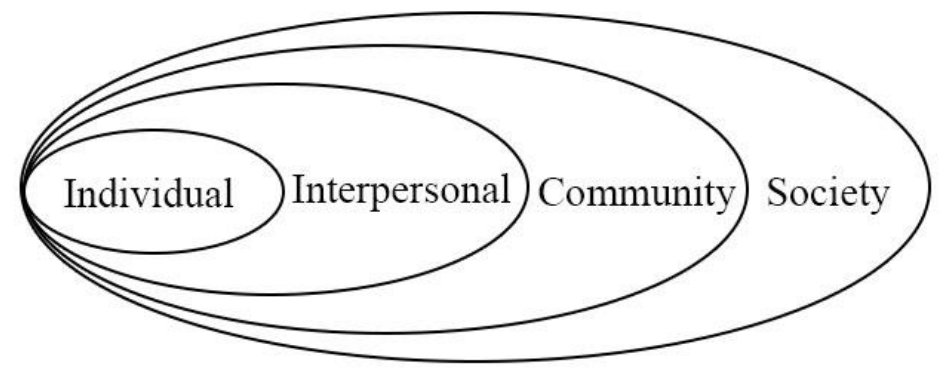

Figure 1. Social-ecological Model.

The social-ecological model seeks to explain behavior as well as foster personal and societal behavior change. Due to the comprehensive nature of examining factors at various levels, the social-ecological approach is more likely to achieve sustainable behavioral change than a single intervention (CDC, 2015). Previous uses of the social-ecological include addressing child abuse and youth violence (Garbarino \& Crouter, 1978; Tolan \& Guerra, 1994) as well as violence against women (Heise, 1998), and has more recently been applied to sexual violence (CDC, 2015; National Association of Student Personnel Administrators, 2004). College health professionals have applied the social-ecological model to address health issues such as alcohol, tobacco, and other drug use, as well as HIV, safety, and stress (National Association of Student Personnel Administrators, 2004). Applying the social-ecological model to violence illustrates how a range of factors put people at risk for experiencing violence or protects them from 
experiencing or committing violence (CDC, 2015). It is important to note that previous work has applied the social-ecological model to sexual assault, but not directly addressed consent.

Even though this study focuses on the communicative aspects of consent that take place at the individual and interpersonal levels, it is important to understand how aspects of the community and societal levels affect communication. Applying the social-ecological model illustrates how individual, interpersonal, community, and societal elements both independently and collectively influence consent. At the individual level, this study examines the effects of perceived behavioral control and empathy. The interpersonal level analyzes sex scripts and relationship metacommunication as well as consent communication for sexual minorities (i.e. non-heterosexual and/or non-cisgender individuals). The community level details particular elements of the college experience that influence consent. The societal level examines how rape culture and rape myths as well as popular culture and media affect consent.

\section{Consent at the Individual Level}

For the purposes of this study, the first level of the social-ecological model examined is the individual level. Individual factors are elements of a person's developmental experience as well as biological and personality aspects that influence his/her actions and increase the likelihood of the person becoming a victim or perpetrating violence (CDC, 2015; Heise, 1998). Perceptions, beliefs, and emotions are also taken into consideration at this level (Kaufman, Cornish, Zimmerman, \& Johnson, 2014). Additional examples of individual factors include age, education, income, substance use, or a history of abuse (CDC, 2015). The present study investigates two individual aspects, perceived behavioral control and empathy, which have been shown to have a relationship with consent, aggression, and/or violence. 
Perceived behavioral control. Perceived behavioral control is a behavioral construct that may help provide insight into consent communication. Conceptually, perceived behavioral control describes people's perceptions of their ability to perform a certain behavior and helps account for intentionality as well as actions (Ajzen, 2002). Three factors largely determine behavioral intent: attitude toward the behavior, subjective norms, and perceived behavioral control (Humphreys \& Brousseau, 2010). Perceived behavioral control, also known as locus of control, is a personality component that explains a person's perceived power over his/her behavior (Keefe, 2006). How prepared an individual feels to perform a behavior is key to actually executing the behavior. Since intentions are the motivating force behind behaviors, it is vital to understand how young adults evaluate the establishment of consent (Humphreys \& Brousseau, 2010). In addition, individuals must feel that they possess adequate internal resources to complete the actions required.

Ajzen $(1991,2002)$ introduced perceived behavioral control as a way to predict behavioral intent while incorporating the effects of perceived self-efficacy. This construct includes two components: internal locus and external locus. Internal locus is the belief that what happens to an individual is a direct result of his/her own actions. Those with external locus feel that sources beyond their control, such as fate, chance, luck, or other individuals, determine events. Those with an internal source of control take responsibility for their own behaviors as well as the outcomes, and cannot be easily persuaded by outside points of view. A behavior may be internally controllable when an individual perceives that he/she possess the skills, confidence, and ability to perform the behavior successfully (Armitage, Conner, Loach, \& Willetts, 1999). The likelihood of successful behavioral performance varies due to the perceived controllability 
of performing the behavior. In addition, the stronger a person's intent to engage in a behavior is, the more likely the action is to be accomplished (Ajzen, 1991).

The degree that young adults evaluate and appraise active establishment of consent is key to predicting their intent to engage in consent negotiation through communication (Humphreys \& Brousseau, 2010). Past research shows that university students resist direct negotiations of sexual consent (Humphreys, 2004). Reasons cited include concerns about feeling awkward and/or spoiling the mood. Previous research also identified sexual assertiveness as positively related to perceived behavioral control, indicating that as individuals' openness, communication, and comfort with sexuality increases their perception that they possess the skills necessary to negotiate consent increases (Humphreys \& Brousseau, 2010). However, Humphreys and Brousseau did not examine possible causes for this phenomenon. Previous research also reveals that college students are ill prepared for the sexual situations they encounter in college (Muehlenhard et al., 2016). For these reasons, this study posits:

$\mathrm{RQ}_{1}$ : Do students who received prior consent education differ in their levels of perceived behavioral control as compared to students who did not receive prior consent education? $\mathrm{RQ}_{2}$ : Does how prepared students feel predict perceived behavioral control?

Empathy and consent. Personal attributes such as empathy greatly affect one's communication style and aptitude. Conceptually, empathy is the cognitive ability to understand others' mental and emotional states (Eisenberg, 2000). Individuals use this understanding to assess others' feelings and to make behavioral decisions based on their assessments (Bruneau, 2009). Empathy is an essential component to respectful communication, and practicing empathy improves communication. In addition, empathy helps to develop trust and predictability as well as to foster friendlier and more open communication. Persons who score high in empathy have 
more emotional sensitivity and self-control (Konrath, O’Brien, \& Hsing, 2011; Olderbak, Sassenrath, Keller, \& Wilhelm, 2014). This is important because emotions guide information processing and decision making (So et al., 2015).

College health and mental health professionals note differences in today's college students compared to previous generations. Over the past decade, college students' capacity for empathy has declined by $40 \%$ (Konrath et al., 2011). Researchers theorize numerous reasons for this including reliance on social networking as well as the rise of violence in video games and other electronic mediums (Dolby, 2014; Konrath et al., 2011). This lack of face-to-face communication is an important consideration for the current generation of college students (Turkle, 2011). As individuals become more distant, empathy declines (Dolby, 2014). An increased focused on one's self leads to a decline in the emphasis on others (Konrath et al., 2011). The current college-aged population even avoids talking on the phone (Turkle, 2011). Empathy is vital to interpersonal communication. Empathy assists in the effective navigation of the social world (Konrath et al., 2011; Olderbak et al., 2014). In addition, empathy allows individuals to relate to others in a way that promotes cooperation and unity rather than conflict. Empathy is an important component to successful social interactions that contributes to an individual's ability to understand and adapt to another person's emotions (Spreng, McKinnon, Mar, \& Levine, 2009). Thus, empathy assists individuals with engagement in the successful communication of emotions and prosocial behavior.

While empathy has not been examined in relation to consent, previous research does link empathy to sexual aggression (Thorton, Todd, \& Thornton, 1996). Research frequently links empathy to aggression because of empathy's suppressing effects on aggression (Vachon, Lynam, \& Johnson, 2014). Empathetic individuals use information about others' affective states to avoid 
potentially harmful behavior as well as to be able to recognize and feel concern when someone else is in distress. Empathetic people also self-correct, recognizing and correcting when their own behavior is aggressive. For these reasons, the following research questions and hypothesis are presented:

$\mathrm{RQ}_{3}$ : Is empathy related to perceived behavior control in the context of sexual consent?

$\mathrm{H}_{1}$ : Empathy positively correlates with attitudes toward establishing consent.

$\mathrm{H}_{2}$ : Empathy positively correlates with consent norms.

\section{Consent at the Interpersonal Level}

The second level of social-ecological model is the relationship or interpersonal level. This level examines close relationships, such as couples, family, and close peer groups (Kaufman et al., 2014). These close relationships greatly influence individuals, and may increase the likelihood of an individual experiencing or committing violence (CDC, 2015). This section examines the communicative aspects of consent as well as sex scripts and relationship metacommunication.

Communicating consent. The ability to successfully communicate consent is a crucial component to healthy and equitable sexual relationships (Cowley \& Reynolds, 2004; Hust et al., 2014). Consent is of particular concern for the college-aged population, even outside of the high rates of sexual assaults on college campuses. The current generation of college students, Millennials, tend to avoid face-to-face communication and lack interpersonal skills (Dolby, 2014). This trend carries over to avoiding direct conversation regarding consent, with young adults relying on indirect, passive nonverbal approaches to communicate in sexual situations (Humphreys, 2004; Humphreys \& Brousseau, 2010; Johnson \& Hoover, 2015; Jozkowski et al., 
2014; Lim \& Roloff, 1999). Alarmingly, young adults consider a lack of response or resistance to constitute consent (Hickman \& Muehlenhard, 1999; Johnson \& Hoover, 2015).

Even though there is discord in defining consent, researchers tend to agree that consent is generally granted in one of three ways: as a cognitive act without any overt communication also known as internal state of willingness, indirectly communicated through actions and/or unclear verbal statements, or directly communicated through verbal statements (Hickman \& Muehlenhard, 1999; Humphreys \& Brousseau, 2010; Muehlenhard et al., 2016). An internal state of willingness implies that consent is an inner state and that partners make inferences based on observable behavior (Muehlenhard et al., 2016). This type of consent is problematic because internal states are private and unknowable without communication. In addition, wanting to have sex and consenting to have sex are not the same thing. Someone can want to do something, sexual in nature or not, but not be willing to do it. Discrepancies between wanting to have sex and being willing to have sex are common.

Lim and Roloff (1999) identified three ways that consent is typically communicated: affirmative non-consent, affirmative behavior, and affirmative language. Affirmative language requires permission be requested and then verbally granted in an unambiguous and positive manner by the partner. However, such straightforward methods are rarely used and often individuals rely on ambiguous nonverbal signals. Without a clear, verbal statement, ambiguity exists as to whether consent is granted. Clear, straightforward verbal communication goes against the sexual scripts of the college-aged population (Humphreys \& Brousseau, 2010).

However, relying on nonverbal signals to communicate consent is highly problematic. Nonverbal cues are vague, ambiguous, and vary in the degree that they communicate consent (Humphreys \& Brousseau, 2010; Lim \& Roloff, 1999). Affirmative consent language requires 
the initiator to ask permission to engage in a specific sexual activity and dictates that the receiver must verbally respond in an unambiguously positive manner before the action can continue. However, such directness is rarely enacted (Lim \& Roloff, 1999). Often sexual activity precedes verbal consent. Without a clear verbal statement of consent, ambiguity exists. Even though affirming language standards seek to clarify sexual communication, sex differences in interpretation may still exist. Verbal communication provides a clearer expression of consent than nonverbal cues; however, nonverbal cues are most often used. While the nonverbal sequence does help inform one's partner, the presence of verbal statements significantly increases the clarity of consent.

Another factor that complicates consent communication is that men and women communicate in different ways. Sex differences exist in how men and women communicate consent. Some men have reported utilizing deceptive techniques and behaviors as their mechanism to indicate consent (Jozkowski \& Peterson, 2013). Nearly 13\% of men indicated that they would communicate consent to vaginal-penile intercourse by inserting their penis into the woman's vagina and pretend it happened by mistake. Lack of mutually agreed upon communication complicates consent (Johnson \& Hoover, 2015). Men are more likely to use nonverbal initiation signals (Hickman \& Muehlenhard, 1999); whereas, women are more likely to use indirect verbal signals, such as asking their partner if they have a condom. And, men are more likely to use indirect nonverbal signals, such as touching and kissing. Both men and women report that they most often communicate their consent by making no response. Men and women report rarely using direct refusal to communicate consent. Men are more likely to use nonverbal cues to indicate consent or non-consent, while women are more likely to use verbal 
cues. Women are also more likely to use a combination of verbal and nonverbal cues (Jozkowski et al., 2014).

Despite existing research, confusion and ambiguity still exists concerning what males and females interpret as sexual consent communication from their partner (Lim \& Roloff, 1999). Previous research indicates that men often inaccurately evaluate women's sexual interest, misinterpreting friendliness for sexual attraction or interest (Lindgren et al., 2008). Women and men may expect their partner to consent in the same way they themselves would signal consent, leading to possible gender-based misunderstandings (Hickman \& Muehlenhard, 1999). In addition, men are more likely to assume consent based on nonverbal cues (Jozkowski et al., 2014).

Young adults' sexual communication does not include elements of verbal, affirmative consent that is the standard for most legal and university requirements for consensual experiences. College students report interpreting lack of response or lack of resistance as consent to sexual activity (Humphreys \& Brousseau, 2010). Young heterosexual adults often do not engage in verbal or direct methods of establishing sexual consent (Johnson \& Hoover, 2015). Because consent communication and interpretation often utilizes ambiguous, nonverbal cues, even well intentioned partners can be at risk for misreading their partners' interest (Hall, 1998; Hickman \& Muehlenhard, 1999; Jozkowski, 2011). These actions are not necessarily due to a lack of understanding. Jozkowski, Peterson, Sanders, Dennis, and Reece (2014) found that even though college students defined consent as explicit communication of an agreement for sexual behavior, this is not how they communicate consent.

This disconnect between consent communication and interpretation exists for a variety of reasons. First, there may be discrepancies in what college students say they do and what they say 
their partners do in sexual situations (Jozkowski et al., 2014). Individuals might believe that they communicate consent verbally, but in actual sexual situations they fall back on commonly used unclear, nonverbal indicators. In addition, even when verbal indication is given, it may be tentative or indirect - which is difficult for partners to interpret. Second, Jozkowski et al. found sex scripts influence college students' sex communication. Participants also reported feeling uncomfortable asking their partners for explicit verbal consent, believing that it ruins the mood or that consent is implied unless otherwise specified.

Since consent is often communicated using ambiguous, nonverbal cues, even well intentioned partners are at risk for misreading signals (Hickman \& Muehlenhard, 1999; Jozkowski, 2011; Jozkowski \& Peterson, 2013). Substantial literature exists that suggests miscommunication about sexual consent may contribute to sexual aggression (Jozkowski et al., 2014). More concerning is the fact that young adults often report that they believe they are able to accurately read their partner's signals (Johnson \& Hoover, 2015). However, if no clear verbal consent statements are uttered, ambiguity exists (Lim \& Roloff, 1999). Research shows that instead of stopping to assess ambiguity, individuals - particularly males - proceed (Jozkowski \& Peterson, 2013). Young adults cite one of the reasons they do this is to avoid embarrassment (Humphreys, 2004; Humphreys \& Brousseau, 2010; Jozkowski et al., 2014). When they perceive that their partner would negatively react to their communication, they are less likely to exhibit consent behaviors (Johnson \& Hoover, 2015).

Another factor that complicates consent communication is the fact that it changes as the nature of the relationship between sexual partners evolves. Relationship status and consent behaviors influence each other (Hickman \& Muehlenhard, 1999; Jozkowski \& Peterson, 2014). The longer a couple is in a relationship, the less perceived need there is to ask for consent before 
engaging in sexual activity (Humphreys, 2007). However, sexual aggression can still occur in a dating context (Spence et al., 1991; Struckman-Johnson, 1988). Thus, the length of a relationship also affects the formality of consent negotiations.

Lastly, differences in consent communication also exist in relation to the sexual activity that takes place. Individuals employ different cues for different types of sexual activity (Jozkowski et al., 2014). For example, verbal cues or a combination of verbal and nonverbal cues are more likely to be used for what are perceived as more intimate behaviors such as vaginalpenile and anal intercourse. A hierarchy of behaviors also exists in that the more individuals perceive an act as intimate, the more likely they are to obtain consent (Humphreys, 2007). Individuals report that penetrative sexual acts such as oral, vaginal, or anal intercourse require more explicit consent than other behaviors that are perceived as less intimate such as kissing and touching (Humphreys, 2004).

Sex scripts. Traditional sex scripts largely influence consent communication and help explain some of the gender-based differences in consent situations. Sex scripts are cognitive frameworks that instruct individuals about how to understand and act in sexual situations (Gagnon, 1990; Gagnon \& Parker, 1995; Masters, Casey, Wells, \& Morrison, 2013). Sex scripts represent the normative order of events that society tells us take place during sexual encounters (Sakaluk, Todd, Milhausen, Lachowsky, \& Undergraduate Research Group in Sexuality, 2014). Sexual scripts are important because they describe normative cultural expectations and behaviors surrounding sexual experiences (Jozkowski \& Peterson, 2013). Consequently, sex scripts exist on a cultural level and the individual level (Humphreys \& Herold, 2007). Cultural sex scripts help comprise mainstream gender norms for heterosexual sexual activity. The majority of sex script research privileges traditional heterosexual sexual situations between one man and one 
woman. Exposure to dominant heterosexual scripts is negatively associated with consent negotiation intentions (Hust et al., 2014).

Alarmingly, consent is not a part of the traditional heterosexual sex script (Hickman \& Muehlenhard, 1999). Token resistance is one aspect of the traditional heterosexual sex script that states women say no to sex when they really mean yes (Muehlenhard \& Hollabaugh, 1998). Women report doing this for a variety of reasons, including practical reasons such as not wanting to appear promiscuous, inhibition-related reasons including religious or moral concerns, and manipulative reasons such as game playing. College students report high acceptance of token resistance (Muehlenhard et al., 2016); however, token resistance is highly problematic. Token resistance increases an individual's risk for sexual victimization (Krahé, Scheinberger-Olwig, \& Kolpin, 2000). Accepting token resistance as part of the sex script is linked to sexual aggression in males. This is due to men interpreting refusal as token resistance, leading to nonconsensual sexual activity (Jozkowski \& Peterson, 2013). In addition, token resistance can contribute to sexual miscommunication (Lim \& Roloff, 1999).

Despite increased efforts toward gender equity and increased rape education, collegeaged individuals still adhere to traditional beliefs about sexual roles (Jozkowski \& Peterson, 2013). In traditional sexual experience gender roles, men play the role of sexual initiators and women playing the role of sexual gatekeepers who ultimately make the decision of whether sexual activity occurs. Additionally, in traditional sex scripts women's pleasure is secondary to men's and adoption of the traditional heterosexual script contributes to rape-supportive social environments. Previous research shows that both men and women reinforce traditional roles for both themselves and the opposite gender (Jozkowski \& Peterson, 2013; Masters et al., 2013). Changing sex scripts to embrace equality for both partners can lead to increased sexual 
satisfaction, safety, and well-being (Masters et al., 2013). However, new sex scripts do not emerge on their own (Laumann \& Gagnon, 1995). Cultural shifts in sex script norms begin at the individual and interpersonal level.

Unfortunately, existing sex script and consent research largely focuses on heterosexual interactions. While, in some instances, school-based sexuality education is becoming more inclusive of LGBTQ+ individuals (Gowen \& Winges-Yanez, 2014), little is known about the sex scripts and consent communication of those with minority sexual orientations and/or gender identities. This is problematic for many reasons, including the fact that the LGBTQ community is at higher risk for sexual assault and less likely to report assaults to formal sources (Long, Ullman, Long, Mason \& Starzynski, 2007; White House Council on Women and Girls, 2014). In one of the few published studies on sexual minorities, Beres, Herold, and Maitland (2004) sought to identify behaviors that individuals would use to ask for and indicate sexual consent to same-sex partners. Survey results from 257 participants found no significant differences in initiating behaviors by men who have sex with men as compared to women who have sex with women. When responding to initiating behaviors, Beres et al. found that men who have sex with men reported using significantly more nonverbal responses than women who have sex with women. However, this study did not capture data beyond behaviors, such as perceptions or attitudes, and failed to capture information on non-traditional gender identities.

$\mathrm{RQ}_{4}$ : Do groups that have different sexual identities differ on attitudes towards establishing consent, approaches to establishing consent, and consent norms? It is important to recognize consent as a gendered construct. Heterosexuality exists due to the social construction of masculinity and femininity (Corteen, 2004). With the expansion of 
gender to include identities outside of the male-female binary, it is imperative that consent research expand to do the same.

RQ5: Do individuals with different gender identities differ on attitudes towards establishing consent, approaches to establishing consent, and consent norms?

Metacommunication. Communication is a vital component of all relationships.

Communication defines relationships and implies commitment (Watzlawick, Beavin, \& Jackson, 1967). Metacommunication, or partner communication about how the relational partners communicate, encompasses conversations about the nature of the relationship between the individuals in the relationship (Knobloch, Haunani Solomon, \& Theiss, 2006; Satir, 1967). Metacommunication occurs when partners give verbal and nonverbal signals about how messages should be understood (Galvin, Braithwaite, \& Bylund, 2016). The ability to metacommunicate is an important aspect of successful communication and results in an awareness of others (Watzlawick et al., 1967). Relationship metacommunication is important and closely tied to the well-being of individuals, the vitality of relationships, and feelings of equity (Knobloch et al., 2006).

Communicating consent is much more complex than simply saying "yes" to sexual initiation. Ideally, consent is an ongoing communicative process and negotiation between partners that continues throughout and beyond the sexual encounter (Reynolds, 2004). When partners do engage in metacommunication, they rarely discuss physical intimacy (Baxter \& Wilmot, 1984). Little research exists about how college students communicate consent with their partners in sexual situations and beyond.

What scholarly research does exist suggests that communication about physical aspects of a relationship does not occur frequently. In an ethnographic study of relational 
metacommunication and relationship turning points, Reherman (1987) conducted eight interviews with relational partners. Of the 352 turning points identified, only 18 dealt with physical intimacy components such as kissing, lust/physical attraction, or sex. Reherman noted that, in particular, the physical attraction turning point involved very little metacommunication. In addition, less than five percent of responses involved expressing expectations regarding relationships in any capacity.

In another study, Miller-Ott and Linder (2014) found that individuals experience discomfort when discussing sexual issues with their partners. Participants in their study often reported using face-saving and humor to help them navigate difficulties surrounding sexual communication. Miller-Ott and Linder found these two verbal communication strategies helpful for sexual partners to communicate in a respectful manner that maintains the integrity of the relationship. Additionally, utilizing humor and face-saving strategies allowed sexual conversations to occur in a non-threatening manner.

College health professionals must understand how college students communicate about physical intimacy with their partners in order to create realistic and effective consent initiatives. Messages that are currently reinforced by consent education, such as no means no and yes means yes, tend to imply that consent is a one-shot agreement. In reality, consent negotiation should continue throughout the entire sexual encounter and beyond (Cowling \& Reynolds, 2004). While metacommunication and ongoing negotiation have been measured in previous studies, researchers have not examined a link between the two constructs. For this reason, the following question is posed:

$\mathrm{RQ}_{6}$ : Does willingness to engage in metacommunication predict better recognition of consent as an ongoing process? 


\section{Consent at the Community Level}

Now that we considered individual and interpersonal aspects of consent, we move on to the third level of the social-ecological model. The community level includes group influences on a larger scale, taking into consideration the community's specific characteristics and norms (Kaufman et al., 2014). Communities in the context of the social-ecological model are any public setting that involves many individuals where social relationship occur such as schools, workplaces, and neighborhoods (CDC, 2015). At this level, the social-ecological model seeks to identify characteristics of settings that are associated with experiences or perpetrating violence. For the purposes of this study, community refers to colleges and universities.

Unique challenges of college campuses. While the risk of sexual assault is not limited to the college population, college students often engage in behaviors such as alcohol use that can complicate securing consent. Due to the propensity for college students to engage in sexual activity while under the influence, alcohol is a large part of college students' sexual experiences (Ward, Matthews, Weiner, Hogan \& Popson, 2012). Experts estimate that nearly three quarters of college sexual assaults take place when the victim is incapacitated by alcohol and/or drugs (Gray, 2014). Previous research also links increased alcohol usage to an increased risk of sexual victimization, particularly for women. Sexually aggressive acts that occur between acquaintances and partners and/or those that occur within normal social settings such as a party are less likely to be labeled as rape by victims (Hersh \& Gray-Little, 1998). Such crimes are also less likely to have legal consequences for the perpetrator.

It is dangerous to assume that college sexual assaults occur because of miscommunication or poor decision making due to alcohol. In fact, the college perpetrator looks startlingly similar to any other sexual predator. Lisak and Miller (2002) interviewed nearly 2,000 
male college students over a 20-year period. They found that one in 16 participants had committed acts that would fit the legal definitions of sexual assault and rape; however, they were never charged or convicted. The participants did not consider themselves rapists, but had committed an average of six assaults each. The researchers' findings echo national statistics that repeat offenders account for nine out of 10 rapes (Lisak \& Miller, 2002). In addition, the participants that had indicated sexual aggression had also committed other acts of interpersonal violence, such as battery and child physical and sexual abuse. Such participants likely escaped legal prosecution because of their choice of victim. Often, participants' victims were acquaintances within the perpetrators social network, and acquaintance rape is less likely to be reported (Lisak \& Miller, 2002). Additionally, the crimes described by the offenders in Lisak and Miller's study likely did not produce any visible injuries, which are crimes that are less likely to be prosecuted. Lastly, due to the use of alcohol and other factors the accused could claim the victim consented which makes a victim less likely to report the crime.

In order to address some of the unique circumstances that college students experience, many institutions of higher education have created prevention programs targeting sexual violence and policies around the concept of consent. Prevention programs include educational programs such as facilitated lectures and workshops as well as educational materials designed to increase awareness (Beres, 2014). These interventions have demonstrated outcomes such as increased awareness of rape myths, increased empathy for victims, increased sexual assault awareness, and increased bystander behavior (Bradley, Yeater, \& O’Donohue, 2009; Foubert, Godin, \& Tatum, 2010; Hanson \& Gidycz, 1993). However, very few measure programming’s effect on consent intentions and behaviors. 
The effectiveness of current college consent initiatives is questionable. Young adults often do not engage in the direct, verbal communication that affirmative consent programs promote (Johnson \& Hoover, 2015; Reynolds, 2004). Rape myths and sociocultural forces are in direct conflict with obtaining verbal affirmative consent or talking directly about sexual desires at all. In addition, current sexual assault prevention education efforts do not include many indirect and nonverbal strategies that students view as indicators of consent (Jozkowski \& Peterson, 2014). Most education and awareness campaigns stress the importance of consent, but do not clarify what constitutes consent (Muehlenhard et al., 2016).

Poor sexual preparation of college students. Existing research shows that today's college students are not prepared to successfully navigate sexual situations that they experience at college. Their limited knowledge about sex adds to the complexity of consent on college campuses (Muehlenhard et al., 2016). There are a few reasons for college students' lack of knowledge and poor preparation. First, the majority of U.S. high schools provide abstinence-only education instead of comprehensive sex education (Muehlenhard et al., 2016). Abstinence programs tend reinforce gender stereotypes and disseminate inaccurate information about contraception (Kantor, Santelli, Teitler, \& Balmer, 2008). College programs tend to not be inclusive, and abstinence-only programs do not address how to give, ask for, or judge sexual consent (Muehlenhard et al., 2016). Lack of knowledge about sex combined with popular culture messages that students are exposed to regarding the negotiation of consent lead many students to be ill prepared for sexual situations they encounter at college. Thus, the following research question is posed:

$\mathrm{RQ}_{7}$ : Does prior consent education affect approaches to establishing consent, attitudes towards establishing consent, consent norms, and ongoing consent communication? 


\section{Consent at the Societal Level}

To fully understand the elements that affect college students' consent communication, it is important to look beyond campuses and consider societal influences. The societal level of social-ecological model examines broad societal factors that assist in creating a climate that encourages or represses violence (CDC, 2015). Factors at this level include social and cultural norms that support violence as well as health, economic, educational, and social policies that reinforce and maintain inequities. Aspects of society that affect consent include rape culture and the portrayal of sex in media and popular culture.

Rape culture and rape myths. The social construction of consent affects consent communication and behaviors. The exact opposite of a consent-focused culture is rape culture. Rape culture is a belief system that supports and normalizes sexual violence throughout a particular society (Guckenheimer, 2008; Maxwell, 2014). Rape-supportive environments increase risk factors related to sexual violence (Buchwald, Fletcher, \& Roth, 1993). Rapesupportive societies foster and encourage rape by teaching that sexual aggression is natural and normal, with males typically as the aggressor (Herman, 1984). Rape culture theorists argue that gender-based sexual violence is not natural behavior, but learned (Guckenheimer, 2008). In rapesupportive societies, sexual-based violence not only becomes the norm - it becomes sexy. Rape culture serves as a link that connects gender, socialization, media, and institutions.

Rape culture leads to many negative personal and societal outcomes. A rape-supportive society influences risk factors related to sexual violence, fosters silencing, and influences postrape behaviors (Guckenheimer, 2008). Aspects of rape culture also contribute to the gross underreporting of sexual assaults. This leads to the concealment of rape and causes a rapesupportive society, further perpetuating sexual violence (Burnett et al., 2009). A rape-supportive 
society often casts doubt and blames victims for their assaults (Guckenheimer, 2008). Factors such as clothing, alcohol or drug use, and visiting particular locations are some of the common reasons rape cultures attribute to a woman provoking a man into raping her. Alcohol and college sexual assaults have a particularly tenuous relationship. Approximately half of sexual assaults involve alcohol use by the perpetrator, victim, or both (Abbey, Zawacki, Buck, Clinton, \& McAuslan, 2016). Alcohol use at the time of the assault makes victims less likely to report the crime out of fear that others may perceive the rape as the victim's fault (Fisher, Daigle, Cullen, $\&$ Turner, 2003). In fact, less than half of rape survivors consider themselves legitimate victims of sexual assault.

The foundation of rape culture lies in rape myths. Rape myths are the normative ideas that are prevalent in rape-supportive cultures (Burnett et al., 2009). Rape myths are social and cultural phenomenon that form social norms that make rape myths seem like normal belief and behavior patterns, further perpetuating the myths. Several rape myths are reoccurring and prominent in rape-supportive culture, covering a wide variety of topics from skepticism of rape claims to excuse certain behaviors and attitudes as boys being boys (Boux \& Daum, 2015). Additional prominent rape myths include: accepting sexual aggression as normal and that female resistance to sex is a normal part of sexual encounters, asserting that victims cause their assaults by engaging in promiscuous behavior, claiming that women falsely report rape to protect their reputations or to retaliate, and that real rape is only perpetrated by a stranger.

Rape myths are particularly concerning because they shape problematic sexual communication (Johnson \& Hoover, 2015). There are three rape myths that are central to consent. The first is that unintentional sexual activity occurs. The second is that miscommunication about sexual activity happens. Lastly, rape cannot occur in an existing sexual 
relationship. Coercion and lack of consent causes most reported accidental or unintentional intercourse. In fact, the most commonly accepted rape myth among college students is that the perpetrator did not intend to commit an assault (Vandiver \& Rager Dupalo, 2012). This assumes that sexual assault occurs because of a harmless miscommunication, and seeks to excuse the perpetrator's behavior while blaming the victim (Derning et al., 2013; Vandiver \& Rager Dupalo, 2012).

Previous research details the pervasiveness of the concept of consent miscommunication in the sexual assault narrative. Koss, Dinero, Seibel, and Cox (1988) found that 59\% of women raped by a casual or steady dating partner characterized the assault as a miscommunication, whereas only $21 \%$ of women attacked by a stranger implicated poor communication. In addition, Muehlenhard and Linton (1987) found that in instances of dating sexual aggression $40 \%$ of victims reported the perpetrator felt led on and $51 \%$ of men reported having engaged in sexual aggression because they felt seduced.

Effects of popular culture and media. Since the 1960's, the portrayal and discussion of sex by popular culture and media sources have become increasingly explicit (Reynolds, 2004). Media sources expose students to copious amounts of messages that suggest communication, negotiation, and equality are unnecessary or impossible in sexual situations (Hust et al., 2014; Reinholtz, Muehlenhard, Phelps, \& Satterfield, 1995). These information sources are important because previous research found them to be a greater influence on sexual intentions than school and religion (L'Engle, Brown, \& Kenneavy, 2006). The effects of media on socialization is different from the influence of parents and school because individuals choose what media sources to consume (Hust \& Brown, 2008). In addition, popular culture and media promote and reinforce traditional sex scripts as well as token resistance (Sprecher et al., 1994). 
Media effects theories explain how repeated exposure to media messages leads individuals to adopt certain opinions and behaviors. When people are repeatedly shown sexual situations that do not involve consent communication or counter consent, they embrace these as truths (Werder, 2009). Previous research also identifies that media consumption affects individuals' beliefs and attitudes about sexual assault (Hust et al., 2013). In addition, repeated media consumption correlates with aggressive behavior.

Due to the multitude of information sources that college students are exposed to, the following research question is posed:

$\mathrm{RQ}_{8}$ : From what sources do college students report getting information about consent? Applying the social-ecological model to consent illustrates the complex factors at play that ultimately influence consent norms and behaviors. Examining elements at the individual, interpersonal, community, and societal levels allows for better understanding of how college students communicate consent. While the ultimate goal is to improve ethical consent decisions and communication between individuals in sexual encounters, improving consent communication at the individual and interpersonal levels can effect consent at the societal level, ushering in a shift from rape-supportive culture to a consent-supportive culture that values equality and pleasure for all individuals. 


\section{CHAPTER II: METHOD}

To study consent communication, behaviors, norms, and the role of empathy in college students, the present study analyzed quantitative data collected via an online survey utilizing multiple statistical procedures. Participants responded to demographic and screening questions as well as seven scales, reporting on their own attitudes and behaviors regarding sexual consent.

\section{Participants}

A total of 295 students at a large Midwestern university responded to the online survey. Participants were overwhelming female $(n=217 ; 73.6 \%)$, followed by males $(n=74 ; 25.1 \%)$. Two students identified as agender and two respondents reported being fluid/genderqueer, meaning $1.4 \%$ of respondents identified as gender non-conforming. The majority of respondents were heterosexual (81\%), followed by bisexual (8.1\%), asexual (5.4\%), gay/lesbian $(3.4 \%)$, pansexual (1.4\%), and questioning/unsure (.7\%).

More than half of participants reported being in a committed relationship with one sexual partner $(52.9 \%)$. Just over a quarter reported that they were not currently sexually active, but have been in the past. Additional responses reported that they had multiple non-committed sexual partners $(7.8 \%)$ or one non-committed sexual partner $(7.1 \%)$. Just over $5 \%$ reported that they had never been sexual active. For those that responded they were in a committed relationship with one sexual partner, the average number of months was $31.13(S D=30.03)$ with a range of 1 to 100 months.

The mean age was 23.99 years old $(S D=6.99)$, with a range from 18 to 72 . The majority of respondents were fourth year students (43.4\%), followed by graduate students $(24.1 \%)$, third year $(18.6 \%)$, and second year $(12.2 \%)$. One respondent was a first year student and three respondents had unique academic standings that did not fall within the traditional years of 
school. Participants primarily identified as Caucasian/White (83.4\%), with the remainder identifying as African American/Black (5.8\%), Hispanic/Latino(a) (5.1\%), Asian (3.4 \%), Asian (3.4\%), and multiracial (2\%). One participant did not wish to disclose this information.

\section{Procedures}

Student participants were recruited utilizing a combination of convenience, random, and snowball sampling. Students were chosen at random to receive a recruitment message through their university email account. Reminder messages were sent out two and four weeks after the initial recruitment message. Due to low response rates from the random sample recruitment message, convenience sampling was employed by sending the recruitment message to all students taking a summer course as well as by utilizing the School of Communication Research Board that lists opportunities for students to participate in research to earn either course credit or extra credit, depending on instructor syllabi policy. In an attempt to make sure that data includes sexual minorities, the researcher shared a recruitment message with LGBTQ+ registered student organizations through email, social media, and face-to-face communication to employ snowball sampling procedures.

In order to participate, students who received recruitment messages had to be 18 years of age and positively respond to the informed consent message. Prior to accessing the survey, participants were told that the survey explores factors that affect sexual consent. The informed consent message included information on the confidentiality of responses, trigger warning related to the content with contact information for campus assistance, and the voluntary nature of participation. After agreeing to informed consent electronically, participants were then directed to the questionnaire. 


\section{Measures}

This study's survey instrument included a total of 78 questions. Five demographic questions collected information on participants' age, year in school, gender they identify with, sexual orientation, and race/ethnicity. Three questions collected information on participants' sexual relationship status, long they have been in their current relationship, and frequency of engaging in sexual activity while under the influence. Three questions created specifically for the present student capture participant's sex education history, collecting information on the inclusion of consent in sex education as well as a ranking of seven consent information sources in relation to the source's level of influence. Two questions assess participants perceived level of preparedness to navigate consent situations and to talk about consent with their current sexual partner. Seven scales collected information on the variables of Empathy, Perceived Behavioral Control, Attitudes toward Establishing Consent, Approaches to Establishing Consent, Consent Norms, Ongoing Negotiation, and Metacommunication. To review the full survey instrument, see the Appendix.

\section{Empathy}

Spreng et al.'s (2009) 16-item Toronto Empathy Questionnaire was utilized to measure participant levels of empathy. Participants responded on a 5-point Likert scale with response options ranging from 1 (strongly disagree) to 5 (strongly agree). Higher mean scores indicate greater levels of empathy. For the purposes of this study, the Toronto Empathy Questionnaire is referred to as empathy. Questions in this scale included "It upsets me to see someone being treated disrespectfully," "I find that I am 'in tune' with other people's moods," "I am not really interested in how other people feel," and "When I see someone being taken advantage of, I feel kind of protective towards them." 
During initial scale development, Spreng et al. (2009) found sound reliability ( $\alpha=.87$ ), unidimensionality for scale confirmed through factor analysis, and strong validity evidence through factor loadings and eigenvalues. Since the Toronto Empathy Questionnaire has not been used extensively, factor analysis and alphas were run again and confirmed sound scale construction. Empathy loaded an acceptable one-factor solution that accounted for $63 \%$ of variance with a 10.09 eigenvalue. Even though one item loaded with less than desirable findings, this item was kept in order to use the scale intact. For the present study, very strong reliability was also confirmed $(\alpha=.96)$.

\section{Perceived Behavioral Control}

The perceived behavioral control subscale from Humphreys and Brousseau's (2010) Sexual Consent Scale Revised was included in the survey instrument. The perceived behavior control scale is 11 items. This scale included questions such as "I am worried that my partner might think I'm weird or strange if I asked for sexual consent before starting any sexual activity," "I believe that verbally asking for sexual consent reduces the pleasure of the encounter," and "I feel confident that I could ask for sexual consent from a new sexual partner." Due to the directional nature of Humphreys and Brousseau's questions, participants responded on a 5-point Likert scale with response options ranging from 1 (strongly agree) to 5 (strongly disagree). For the present study, polarity of this scale was switched so that higher mean scores reflect greater perceived behavioral control.

Humphreys and Brousseau's (2010) initial scale creation found strong internal consistency for this scale $(\alpha=.86$ ), and factor analysis results revealed that perceived behavioral control items loaded onto a single factor and demonstrated strong validity. All 11 items from Humphreys and Brousseau's survey instrument were used for this study. For the present study, 
the factor analysis confirmed a unidimensional scale with all items loading at satisfactory levels. Tests for the current study also demonstrated very strong scale reliability $(\alpha=.94)$.

\section{Attitudes toward Establishing Consent}

The positive attitude towards establishing consent subscale from Humphreys and Brousseau's (2010) Sexual Consent Scale Revised was utilized to measure participants' attitudes surrounding the establishment of consent. The attitudes towards establishing consent scale includes 11 items and participants responded on a 5-point Likert scale with response options ranging from 1 (strongly disagree) to 5 (strongly agree). Higher mean scores reflect a positive attitude toward establishing consent. This subscale included questions such as "I believe that asking for sexual consent is in my best interest because it reduces any misinterpretations that might arise," "Most people I care about feel that asking for sexual consent is something I should do," and "Before making sexual advances, I think that one should assume 'no' until there is clear indication to proceed."

All 11 items from the original scale were used in the present study. Initial statistical tests by Humphreys and Brousseau found strong internal consistency for this scale $(\alpha=.84)$, and factor analysis results revealed a unidimensional scale and demonstrated strong validity. These findings were confirmed for the present study. The factor analysis verified that all 11 items loaded on one factor, demonstrating strong validity. Very strong internal consistency was also confirmed $(\alpha=.95)$.

\section{Approaches to Establishing Consent}

The approaches to establishing consent subscale from Humphreys and Brousseau's (2010) Sexual Consent Scale Revised was utilized to measure participants' attitudes about tactics to determine consent. The approaches toward establishing consent scale includes six items and 
participants responded on a 5-point Likert scale with response options ranging from 1 (strongly disagree) to 5 (strongly agree). Higher mean scores reflect positive approaches to establishing consent. This scale included questions such as "Typically I communicate sexual consent to my partner using nonverbal signals and body language," "It is easy to accurately read my current (or most recent) partner's nonverbal signals as indicating consent or non-consent to sexual activity," and "I always verbally ask for consent before I initiate a sexual encounter."

All six items from the original scale were used in the present study. Initial statistical tests by Humphreys and Brousseau (2010) found respectable internal consistency for this scale $(\alpha=$ .76), and factor analysis results revealed a unidimensional scale and demonstrated strong validity. These findings were confirmed for the present study, using principle components extraction and forcing a one-factor solution. The factor analysis verified that all six items loaded on one factor, demonstrating strong validity. Acceptable internal consistency was also found $(\alpha=$ .79).

\section{Consent Norms}

The consent norms scale was constructed for the present study utilizing a modified version of Humphreys and Brousseau's (2010) Sexual Consent Scale Revised to measure how norms affect participants' perceptions of consent. For the present study, the consent norms scale includes eight items and participants responded on a 5-point Likert scale with response options ranging from 1 (strongly disagree) to 5 (strongly agree). Higher mean scores indicate more favorable adoption of positive consent norms. The first seven items in the present study's consent norms scale were taken from Humphreys and Brousseau's consent norms subscale. This scale included questions such as "I think that obtaining sexual consent is more necessary in a new relationship than a committed relationship," "I believe it is enough to ask for consent at the 
beginning of a sexual encounter," and "If consent for sexual intercourse is established, petting and fondling can be assumed." Humphreys and Brousseau found acceptable internal consistency for this scale $(\alpha=.67)$, and the exploratory factor analysis (EFA) revealed a unidimensional subscale and demonstrated strong validity. The last item in the scale of the present study is from a different scale from Humphreys and Brousseau's Sexual Consent Scale Revised: "I have not given much thought to the topic of sexual consent."

Due to the fact that this scale was altered, tests were run to ensure scale validity and reliability. Initial EFA found the scale to load on more than one factor, leading to subsequent EFAs that removed questions $65,62,64$, and 61 in that order. The final EFA produced an acceptable one-factor solution. Both the KMO measure (.763) and Bartlett's test $\left[\chi^{2}=466.835\right.$ (6), $p<.001]$ were acceptable. The remaining four items loaded unidimensionally and demonstrated very good internal consistency $(\alpha=.84)$, that explained $61.00 \%$ of variance with a 2.44 eigenvalue. See Table 1 for factor final loadings.

\section{Ongoing Negotiation}

The ongoing negotiation scale was created by using four items from the sexual consent behaviors scale (Humphreys \& Herold, 2007) and four original items created specifically for the present study. Participants responded on a 5-point Likert scale with response options ranging from 1 (strongly disagree) to 5 (strongly agree). Higher mean scores indicate greater willingness to engage in ongoing negotiation. The four items from the sexual consent behaviors scale were "During a sexual encounter, I typically only GIVE my consent once," "During a sexual encounter, I typically only ASK for consent once," "If a sexual request is made and the partner indicates 'no,' it is okay to continue negotiating the request," and "I tend to NOT decide ahead of time what I will and will not consent to sexually and wait until I am 'in the moment' to decide." 
Reliability for the sexual consent behaviors scale was acceptable $(\alpha=.66)$ and EFA found sound validity evidence for the two factors. Items created for the present study were "During a sexual encounter, I typically GIVE my consent for each new sexual activity," "During a sexual encounter, I typically ASK for consent for each new sexual activity," "During a sexual encounter, I check in with my partner periodically to make sure they are still interested in continuing the encounter," and "I feel comfortable in withdrawing my consent and not continuing at any time during a sexual encounter."

Table 1

Factor Loadings for Consent Norms Scale

Survey Items $\quad$ Loadings

58. I think that obtaining sexual consent is more necessary in a new relationship $\quad .948$ than in a committed relationship

59. I think that obtaining sexual consent is more necessary in a casual sexual $\underline{.879}$ encounter than in a committed relationship

60. I believe that the need for asking for sexual consent decreases as the length $\underline{.654}$ of an intimate relationship increases

63. I believe that partners are less likely to ask for sexual consent the longer they are in a relationship

$\begin{array}{cr}\text { Eigenvalue } & 2.44 \\ \text { \% of Variance } & 61.00\end{array}$

Cronbach's alpha .84

Note. Underlined factor coefficients show acceptable factor loadings for the corresponding items and factors that were retained for statistical analysis. 
Validity and reliability tests were performed because this scale included original items. Initially, the scale loaded on three factors, with many loading values below acceptable range. Subsequent EFA tests eliminated questions 70 and 72, leaving six items that loaded on three factors. Factor one was used for the present study because it had the strongest primary factor loadings and these items closely fit this study's operational definition of ongoing negotiation. Additionally, the two factors made up of Humphreys and Herold questions were not used because these items loaded in a different manner than the original authors used the questions. Even though two is a less than desirable number of items for a scale, this was the best way to proceed due to how the items grouped during factor analysis. The final EFA produced an acceptable one-factor solution that explained $72.88 \%$ of variance with a 1.45 eigenvalue. Both the KMO measure (.500) and Bartlett's test $\left[\chi^{2}=160.759(1), p<.001\right]$ were acceptable. See Table 2 for final factor loadings. The remaining two item scale also demonstrated very good validity $(\alpha=.84)$. 
Table 2

Factor Loadings for Ongoing Negotiation Scale

Survey Items $\quad$ Loadings

68. During a sexual encounter, I typically GIVE consent for each new sexual $\quad \underline{.854}$ activity

69. During a sexual encounter, I typically ASK for consent for each new sexual $\quad .854$ activity

$\begin{array}{rr}\text { Eigenvalue } & 1.45 \\ \text { \% of Variance } & 72.88\end{array}$

Cronbach's alpha .84

Note. Underlined factor coefficients show acceptable factor loadings for the corresponding items and factors that were retained for statistical analysis.

\section{Metacommunication}

The metacommunication scale seeks to assess how participants communicate about consent and sexual activity outside of sexual encounters. This scale was created using items from Humphreys and Brousseau's (2010) and Humphreys and Herold (2007) as well as one original item created specifically for this study. Participants responded to all items on a 5-point Likert scale with response options ranging from 1 (strongly disagree) to 5 (strongly agree). Higher mean scores indicate greater willingness to engage in metacommunication. Three questions in the present metacommunication scale are from Humphreys and Brousseau's (2010) awareness and discussion scale. These items were comprised of "I have discussed sexual consent issues with my current (or most recent) partner at times other than sexual encounters," "I have discussed the topic of sexual consent with a friend," and "I have heard the topic of sexual consent being discussed by other students on campus." The wording of two of these items was 
modified to remove the word issues, in order to avoid insinuating any negative connotation with consent. The four item awareness and discussion scale from Humphreys and Brousseau (2010) had sound internal reliability $(\alpha=.71)$. Additionally, EFA found sound validity for these items. One item from the metacommunication scale is from the consent attitudes scale (Humphreys \& Herold, 2007), “Too few partners openly discuss the issue of sexual consent.” Wording of this item for use in the present study was altered slightly, changing the original use of the word “couples" to "partners" in order to be more inclusive of participants' sexual situations. The item created specifically for the present study stated "I have discussed engaging in sexual activity with my current (or most recent) partner at times other than sexual encounters.”

Because the metacommunication scale was created from multiple sources, reliability and validity tests were performed. All items loaded unidimensionally on one factor and question 78 was eliminated due to poor factor loading. Both the KMO measure (.732) and Bartlett's test $\left[\chi^{2}=\right.$ $422.206(6), p<.001]$ were acceptable. The remaining four items demonstrated strong internal reliability $(\alpha=.84)$ that explained $59.33 \%$ of variance with a 2.374 eigenvalue. See Table 3 for factor loadings of the four retained items. 
Table 3

Factor Loadings for Metacommunication Scale

$\begin{array}{ll}\text { Survey Items } & \text { Loadings }\end{array}$

74. I have discussed engaging in sexual activity with my partner(s) at times other $\quad \underline{.942}$ than during sexual activity

75. I have discussed sexual consent with my partner(s) at times other than sexual $\quad \underline{.819}$ encounters

77. I have discussed sexual consent with a friend

.671

76. Too few sexual partners openly discuss sexual consent

.605

Eigenvalue $\quad 2.374$

$\%$ of Variance 59.339

Cronbach's Alpha $\quad .84$

Note. Underlined factor coefficients show acceptable factor loadings for the corresponding items and factors that were retained for statistical analysis.

\section{Data Analysis}

A variety of statistical tests were used to investigate this study's research questions and hypotheses. Research question one examined whether prior exposure to consent education predicts students' Perceived Behavioral Control. Using perceived behavioral control as the dependent variable, RQ1 was analyzed by conducting an independent samples $t$-test to see if there were any differences between two groups: students who received prior education and those who did not. Research question two sought to assess if student preparedness predicted perceived behavioral control. Because RQ2 examined two continuous predictor variables of preparedness to navigate sexual situations and preparedness to discuss consent with current sexual partner(s) 
in relation to the continuous, independent variable of perceived behavioral control, a multiple linear regression was used to text this research question.

Research question six asked if willingness to engage in metacommunication predicted recognition of consent as an ongoing process. A simple linear regression test was used to see if the continuous predictor variable of metacommunication triggers the continuous outcome variable of ongoing negotiation. Research question three sought to uncover if a relationship exists between empathy and perceived behavioral control in the context of sexual consent. Because there is no existing literature that would suggest a direction for the relationship between these two continuous variables, a correlation test was utilized. Research question eight examined what sources of consent information students rate as influential. This information was collected through a ranking of a variety of information sources and reported with frequency counts.

Research questions four, five, and seven were investigated using multivariate analysis of variance (MANOVA) test for each research question in order to examine multiple levels of variables for main effects. RQ4 sought to determine if the categorical variable of sexual orientation has an effect on the three dependent variables of attitudes towards establishing consent, approaches to establishing consent, and consent norms. RQ5 examines if the categorical independent variable of gender identity's effect on the dependent variables of attitudes towards establishing consent, approaches to establishing consent, and consent norms. RQ7 sought to examine prior consent education's effect on the dependent variables of approaches to establishing consent, attitudes toward establishing consent, consent norms, metacommunication, and ongoing negation. Lastly, RQ8 examined information sources and their levels of influence.

Due to the fact that empathy had not been previously studied directly with sexual consent, this study's two hypotheses were analyzed using correlation tests. H1 sought to 
determine if empathy positively correlates with attitudes toward establishing consent. H2 examines if empathy positively correlates with consent norms. Attitudes toward establishing consent was used as the outcome variable for H1. Consent norms was used as the outcome variable for $\mathrm{H} 2$. Alpha was set at .05 for all statistical tests. 


\section{CHAPTER III: RESULTS}

The previous chapter outlined the methodology employed in the present study. This chapter will detail the results of the hypotheses and research questions proposed in the literature review. Results are organized in relation to the social-ecological model, beginning with results of research questions and hypothesis at the individual level and concluding with results at the societal level.

\section{Consent at the Individual Level}

\section{Perceived Behavioral Control}

The first research question was examined by conducting an independent samples $t$-test to determine if prior consent education affected students' perceived behavioral control. The Levene's test for variance was not significant $(F=2.78, p=.09)$, so equal variance is assumed. Those who received prior consent education did not significantly differ in their reported perceived behavioral control compared to those who did not receive prior consent education $t(238)=.205, p=.83,95 \% \mathrm{CI}[-.24, .30]$. Students that reported receiving prior consent education $(n=163, M=3.71, S D=1.07)$ scores of perceived behavior control did not statistically differ from students who did not receive consent education $(n=77, M=3.74, S D=$ .88). Consequently, results for RQ1 found no significant effect of consent education on perceived behavioral control.

Research question two examined if student level of preparedness predicted perceived behavioral control utilizing a multiple regression. Preparation was measured through two predictor variables examining students' overall perceptions of their preparation to navigate sexual situations and their levels of preparation to talk about consent with their current sexual partner(s). Missing cases were excluded pairwise. Results of the multiple regression analysis 
indicated that preparation accounted for $3.9 \%$ of variance in perceived behavioral control, $R^{2}{ }_{a d j}=$ $.031, F(2,237)=4.772, p=.009$. So while the two predictor variables for preparation predicted a statistically significant amount of variance in perceived behavioral control, the percentage of variance explained was small. Neither of the predictor variables measuring preparation were statistically significant unique predictors of perceived behavioral control. Tolerance and VIF statistics did not indicate the presence of collinearity. Beta weights are reported in Table 4.

Table 4

Beta Weights for Perceived Behavioral Control Model

\begin{tabular}{|c|c|c|c|c|}
\hline Predictor Variables & & $B$ & $S E B$ & $\beta$ \\
\hline Prepared to Navigate Sexual Situations & & .005 & .005 & .088 \\
\hline \multirow[t]{4}{*}{ Prepared to Talk about Consent } & & .008 & .005 & .131 \\
\hline & $R^{2}$ & & .039 & \\
\hline & $R_{a d j}^{2}$ & & .031 & \\
\hline & $F$ & & 4.772 & \\
\hline
\end{tabular}

Note. An * indicates a unique significant predictor variable at $p<.05 .(n=239)$

\section{Empathy}

Hypothesis one predicted that empathy would positively correlate with attitudes toward establishing consent. A bivariate correlation was run to assess the relationship between these two constructs. Empathy demonstrated a very strong, positive association with student attitudes about establishing consent, $r(230)=.78, p<.001$. Thus, higher levels of empathy are associated with positive attitudes toward establishing consent. 
Hypothesis two projected that empathy would positively correlate with consent norms. A bivariate correlation found that empathy demonstrated a moderate, positive association with student consent norms, $r(216)=.55, p<.001$. Consequently, higher levels of empathy are related to students adopting favorable consent norms.

Research question three sought to explore if empathy is related to perceived behavioral control in the context of sexual consent. A bivariate correlation was run to test the relationship and found a very strong, positive relationship between empathy and perceived behavioral control, $r(233)=.74, p<.001$. Therefore, higher levels of empathy are associated with greater perceptions of behavioral control.

\section{Consent at the Interpersonal Level}

\section{Nontraditional Gender Identities and Sexual Orientations}

Research questions four and five explored possible differences between individuals who identify as gender non-conforming and/or with non-heterosexual identities vary from those in the gender and sexual majority. RQ4 asked if those with different sexual identities differ on attitudes toward establishing consent, approaches to establishing consent, and consent norms. Results of the MANOVA test indicated that there were no differences in these variables when controlling for sexual orientation, Wilks $\lambda=.954, F(15,591.16)=.683, p=.80, \eta^{2}=.016$. Additionally, univariate follow-up tests for the groups did not find significant differences for attitudes towards establishing consent, $F(5,216)=1.043, p=.39, \eta^{2}=.024$, approaches to establishing consent, $F(5,216)=.53, p=.74, \eta^{2}=.012$, or consent norms, $F(5,216)=.11, p=.98, \eta^{2}=.003$. Mean differences among all measures were relatively minimal across all sexual identities, with the exception of individuals who identified as bisexuals on the attitudes towards establishing consent scale (see Table 5). 
Table 5

Descriptive Statistics for Sexual Identity

\begin{tabular}{|c|c|c|c|c|}
\hline Sexual Identity & Group & $M$ & $S D$ & $n$ \\
\hline \multicolumn{5}{|c|}{ Attitudes toward Establishing Consent } \\
\hline & Asexual & 3.85 & 1.01 & 14 \\
\hline & Bisexual & 4.22 & .59 & 15 \\
\hline & Gay/Lesbian & 3.65 & 1.35 & 8 \\
\hline & Heterosexual & 3.63 & 1.02 & 181 \\
\hline & Pansexual & 3.63 & .38 & 2 \\
\hline & Question/Unsure & 3.90 & .12 & 2 \\
\hline \multicolumn{5}{|c|}{ Approaches to Establishing Consent } \\
\hline & Asexual & 3.38 & .85 & 14 \\
\hline & Bisexual & 3.02 & .72 & 15 \\
\hline & Gay/Lesbian & 3.04 & .94 & 8 \\
\hline & Heterosexual & 3.27 & .83 & 181 \\
\hline & Pansexual & 3.50 & .70 & 2 \\
\hline & Question/Unsure & 2.91 & .35 & 2 \\
\hline \multicolumn{5}{|l|}{ Consent Norms } \\
\hline & Asexual & 3.62 & 1.08 & 14 \\
\hline & Bisexual & 3.70 & .76 & 15 \\
\hline & Gay/Lesbian & 3.71 & .82 & 8 \\
\hline & Heterosexual & 3.55 & 1.06 & 181 \\
\hline & Pansexual & 3.37 & .53 & 2 \\
\hline & Question/Unsure & 3.75 & .35 & 2 \\
\hline
\end{tabular}

Note. Scores are based on a 5-point Likert type scale from 1 (strongly disagree) to 5 (strongly agree). Higher mean scores indicate greater levels of positive attitudes toward establishing consent, favorable approaches to establishing consent, and positive consent norms.

In order to compare attitudes toward establishing consent, approaches to establishing consent, and consent norms between gender identity groups, a MANOVA test was completed. 
Results of the MANOVA indicated that there was no statistically significant difference in these variables when controlling for gender identity, Wilks $\lambda=.944, F(9,525.838)=1.393, p=.18, \eta^{2}$ $=.019$. There were only a small percentage of respondents identifying as gender non-conforming (i.e., not male or female). However, differences in mean scores were observed for these three scales based on gender identity. See Table 6 for descriptive statistics.

Table 6

Descriptive Statistics for Gender Identity

\begin{tabular}{lllcc}
\hline \multicolumn{1}{c}{ Gender Identity } & \multicolumn{1}{c}{ Group } & $M$ & $S D$ & $n$ \\
\hline Attitudes toward Consent & Agender & 4.54 & .00 & 2 \\
& Female & 3.71 & .99 & 166 \\
& Fluid/GQ & 3.90 & .00 & 1 \\
Attitudes toward Establishing Consent & Male & 3.59 & 1.06 & 53 \\
& & & & \\
& Agender & 2.41 & .35 & 2 \\
Consent Norms & Female & 3.31 & .83 & 166 \\
& Fluid/GQ & 4.00 & .00 & 1 \\
& Male & 3.08 & .76 & 53 \\
& & & & \\
& Agender & 2.75 & .00 & 2 \\
& Female & 3.60 & .99 & 166
\end{tabular}

Note. GQ stands for genderqueer. Scores are based on a 5-point Likert type scale from 1 (strongly disagree) to 5 (strongly agree). Higher mean scores indicate greater levels of positive attitudes toward establishing consent, favorable approaches to establishing consent, and positive consent norms. 


\section{Metacommunication}

A simple regression procedure examined RQ6 to determine if willingness to engage in metacommunication predicted recognition of consent as an ongoing process. Missing cases were excluded pairwise. Results of the simple regression indicated that $2.8 \%$ of the variance in ongoing negotiation could be predicted by metacommunication, $R_{a d j}^{2}=.024, F(1,211)=6.112, p$ $=.01$. Analysis of regression coefficients indicated that metacommunication, $\beta=.168, t=2.472$, $p=.01$, was a statistically significant predictor of consent as an ongoing process. These results indicate that while the results of the regression procedure were significant, metacommunication accounts for only a small amount of variance in consent as an ongoing process. Beta weights for the final regression model can be found in Table 7.

Table 7

Beta Weights for Consent as an Ongoing Process Model

\begin{tabular}{|c|c|c|c|c|}
\hline Variable & & $B$ & $S E B$ & $\beta$ \\
\hline \multirow[t]{4}{*}{ Metacommunication } & & .169 & .068 & $.168 *$ \\
\hline & $R^{2}$ & & .028 & \\
\hline & $R_{a d j}^{2}$ & & .024 & \\
\hline & $F$ & & 6.112 & \\
\hline
\end{tabular}

Note. An $*$ indicates a unique significant predictor variable at $p<.05 .(n=214)$ 


\section{Consent at the Community Level}

\section{Prior Consent Education}

Research question seven explored prior consent education's effect on approaches to establishing consent, towards establishing consent, consent norms, and ongoing consent communication. MANOVA tests were run comparing students who received consent education and those that did not to see if there were any differences on attitudes toward establishing consent, approaches to establishing consent, and consent norms. Results indicated that there were no statistically significant difference in attitudes towards establishing consent, approaches to establishing consent, consent norms, and ongoing communication when controlling for prior consent education, Wilks $\lambda=.991, F(4,202)=.444, p=.77, \eta^{2}=.009$. Mean scores for both groups on all scales were relatively stable as well. See Table 8 for descriptive statistics. 
Table 8

Descriptive Statistics for Prior Consent Education

\begin{tabular}{llccc}
\hline \multicolumn{1}{c}{ Prior Consent Education } & \multicolumn{1}{c}{ Group } & $M$ & $S D$ & $n$ \\
\hline Attitudes toward Consent & Not Selected & 3.73 & .92 & 68 \\
& School/Sex Ed & 3.65 & 1.05 & 139 \\
Approaches toward Establishing Consent & & & & \\
& Not Selected & 3.24 & .78 & 68 \\
& School/Sex Ed & 3.23 & .84 & 139 \\
Consent Norms & & & & \\
& Not Selected & 3.67 & .95 & 68 \\
& School/Sex Ed & 3.50 & 1.07 & 139 \\
Ongoing Negotiation & & & & \\
& Not Selected & 3.14 & 1.04 & 68 \\
& School/Sex Ed & 3.14 & 1.00 & 139
\end{tabular}

Note. Scores are based on a 5-point Likert type scale from 1 (strongly disagree) to 5 (strongly agree). Higher mean scores indicate greater levels of positive attitudes toward establishing consent, favorable approaches to establishing consent, positive consent norms, and increased willingness to engage in ongoing negotiation.

\section{Consent at the Societal Level}

\section{Sources of Influence}

Research question eight sought to collect information on what sources of consent information do students report as most influential. Overall, $82.4 \%$ of participants $(n=288)$ reported that someone had directly discussed sexual consent with them, with $13.9 \%$ saying no one had and 3.7\% reporting that they were not sure. Of those that reported affirmatively that someone had directly discussed sexual consent with them, friend was reported as the most 
influential source. Items in the influence ranking section were recoded so that higher mean scores reflect higher rates of influence. See Table 9 for statistics.

Table 9

Descriptive Statistics for Sources of Influence

\begin{tabular}{lccc}
\hline \multicolumn{1}{c}{ Source of Influence } & $M$ & $S D$ & $n$ \\
\hline Friend & 3.24 & 2.187 & 288 \\
School & 2.81 & 2.099 & 288 \\
Parent(s)/Guardian(s) & 2.80 & 2.27 & 288 \\
Media & 2.60 & 2.10 & 288 \\
Sibling & 1.92 & 2.21 & 288 \\
Religion & 1.70 & 2.25 & 288 \\
\hline
\end{tabular}

Note. Scores are based on a sliding ranking from 1 (least influential) to 7 (most influential). Higher mean scores mean a more influential information source.

\section{Additional Findings}

Supplementary statistical tests beyond those outlined hypothesis and research questions were conducted in order to identify any additional noteworthy results. A bivariate correlation test between all seven scales was conducted in order to make sure they were measuring similar aspects of without collinearity. Attitudes toward establishing consent showed a positive association with empathy, metacommunication, ongoing negotiation, consent norms, and perceived behavioral control. Approaches to establishing consent positively correlated with 
empathy, consent norms, and perceived behavioral control, as well as negatively correlated with ongoing negotiation. See Table 10 for correlation and descriptive statistics.

Although alcohol use is not a large focus of this study, one question did capture information on how often participants engage in sexual activity while under the influence. A bivariate correlation was run to see if a relationship existed between how often students participate in sexual activity under the influence and consent norms. Students engaging in sexual activity while under the influence demonstrated a weak, positive correlation with consent norms, $r(227)=.178, p<.01$ as well as with approaches toward establishing consent, $r(227)=.199, p<$ .01. This positive association with approaches toward establishing consent illustrates that the more often students are engaging in sexual activity under the influence, the more likely they are to feel they are clearly communicating sexual consent as well as perceive themselves to accurately understand their partner's communication about consent.

An additional post hoc tests were run to see if any year in school showed any effect on the variables measured. A one-way ANOVA was performed in order to see if the categorical independent variable of year in school had any effect on each of the continuous variables measured by the survey scales. Results of the ANOVA showed that the only variable that year in school had a significant impact on was consent norms, $F(4,220)=2.644, p=.03$. Even though year in school did not have a statistically significant effect on the remaining six scales, the mean results for all scales trend upward as length of time in school increases. See Table 11 for descriptive statistics of all seven survey scales by year in school.

A one-way ANOVA was also performed to see if there was an interaction effect between relationship type and the continuous variables of empathy, attitudes towards consent, approaches to establishing consent, consent norms, ongoing negotiation, and metacommunication. No 
statistically significant difference was found for these variables based on participant relationship type. Additionally, no discernable pattern in variable means was observed. 
Table 10

Correlations among Scales

\begin{tabular}{|c|c|c|c|c|c|c|c|c|c|}
\hline & 2 & 3 & 4 & 5 & 6 & 7 & $M$ & $S D$ & $n$ \\
\hline 1. Attitudes toward Establishing Consent & -.058 & $.781 * *$ & $.719 * *$ & $.213 * *$ & $.321 * *$ & $.773 * *$ & 3.68 & .99 & 241 \\
\hline 2. Approaches to Establishing Consent & -- & $.187 * *$ & $.180 * *$ & $-.199 * *$ & $.558 * *$ & $.157 *$ & 3.26 & .82 & 227 \\
\hline 3. Empathy & & -- & $.735 * *$ & .091 & $.556^{* *}$ & $.748 * *$ & 3.83 & .88 & 260 \\
\hline 4. Metacommunication & & & -- & $.168 *$ & $.385^{* *}$ & $.674 * *$ & 3.60 & 1.01 & 214 \\
\hline 5. Ongoing Negotiation & & & & -- & -.126 & .112 & 3.13 & 1.02 & 214 \\
\hline 6. Consent Norms & & & & & -- & $.390 * *$ & 3.57 & 1.02 & 227 \\
\hline 7. Perceived Behavioral Control & & & & & & -- & 3.72 & 1.01 & 242 \\
\hline
\end{tabular}

Note. Correlations with an $*$ are significant at the level of $p<.05$, while those with an $* *$ are significant at the level of $p<.01(2$ tailed). Higher mean scores indicate greater levels of positive attitudes toward establishing consent, favorable approaches to establishing consent, higher levels of empathy, greater willingness to metacommunicate, greater willingness to engage in ongoing negotiation, more positive consent norms, and greater perceived behavioral control. 
Table 11

Descriptive Statistics for Scales by Year in School

\begin{tabular}{|c|c|c|c|c|}
\hline Variable & Group & $M$ & $S D$ & $n$ \\
\hline \multicolumn{5}{|l|}{ Empathy } \\
\hline & Second Year & 3.50 & 1.08 & 29 \\
\hline & Third Year & 3.65 & .99 & 53 \\
\hline & Fourth Year & 3.88 & .79 & 111 \\
\hline & Graduate & 4.02 & .79 & 62 \\
\hline \multicolumn{5}{|l|}{ Perceived Behavioral Control } \\
\hline & Second Year & 3.55 & 1.26 & 28 \\
\hline & Third Year & 3.52 & 1.06 & 47 \\
\hline & Fourth Year & 3.80 & .98 & 108 \\
\hline & Graduate & 3.81 & .89 & 54 \\
\hline \multicolumn{5}{|c|}{ Attitude toward Establishing Consent } \\
\hline & Second Year & 3.33 & 1.12 & 29 \\
\hline & Third Year & 3.47 & 1.06 & 46 \\
\hline & Fourth Year & 3.70 & .96 & 106 \\
\hline & Graduate & 3.94 & .86 & 55 \\
\hline \multicolumn{5}{|c|}{ Approaches to Establishing Consent } \\
\hline & Second Year & 3.08 & .76 & 27 \\
\hline & Third Year & 3.24 & .81 & 43 \\
\hline & Fourth Year & 3.22 & .78 & 99 \\
\hline & Graduate & 3.45 & .91 & 53 \\
\hline \multicolumn{5}{|l|}{ Consent Norms } \\
\hline & Second Year & 3.19 & 1.20 & 27 \\
\hline & Third Year & 3.44 & 1.00 & 43 \\
\hline & Fourth Year & 3.63 & .95 & 100 \\
\hline & Graduate & 3.82 & .94 & 52 \\
\hline \multicolumn{5}{|l|}{ Ongoing Negotiation } \\
\hline & Second Year & 2.90 & .93 & 25 \\
\hline & Third Year & 3.28 & 1.05 & 39 \\
\hline & Fourth Year & 3.16 & .99 & 95 \\
\hline & Graduate & 3.15 & 1.08 & 51 \\
\hline \multicolumn{5}{|l|}{ Metacommunication } \\
\hline & Second Year & 3.24 & .96 & 25 \\
\hline & Third Year & 3.47 & 1.11 & 40 \\
\hline & Fourth Year & 3.60 & 1.02 & 94 \\
\hline & Graduate & 3.87 & .09 & 51 \\
\hline
\end{tabular}

Note. Scores are based on a 5-point Likert type scale from 1 (strongly disagree) to 5 (strongly agree). First year is not included because only one first year student completed the scale section of the survey. Higher mean scores indicate higher levels of empathy, greater perceived behavioral control, greater levels of positive attitudes toward establishing consent, more favorable approaches to establishing consent, more positive consent norms, greater willingness to engage in ongoing negotiation, and greater willingness to metacommunicate. 


\section{CHAPTER IV: DISCUSSION}

Successful consent communication is necessary for a mutually enjoyable, respectful, and ethically sound sexual encounter (Cowling \& Reynolds, 2004; Flyntz, 2016; Miller-Ott \& Linder, 2014). While much research has explored the topic of sexual consent, previous studies have largely been conducted by psychology and sociology scholars with an emphasis on nonconsensual situations (Cowling \& Reynolds, 2004). Very little research has focused on the actual communicative components of consent, except to identify that many young adults believe that sexual miscommunication occurs frequently and can sometimes cause sexual assaults (Derning et al., 2013; Johnson \& Hoover, 2015; Vandiver \& Rager Dupalo, 2012). Thus, examining sexual consent through a communication lens is overdue.

The present study focuses on consent as a communicative act, analyzing predictors of college student consent communication. This chapter will provide a synopsis of the present study's outcomes as well as implications for higher education professionals, limitations of the study, and opportunities for future research. The following discussion of results is presented in the order of the social-ecological model, from the interpersonal level to the societal level. While many organizations such as the CDC have used the social-ecological model to classify public health and violence prevention issues, no one has used the model to illustrate the various influences on sexual consent. Applying the social-ecological model helps clarify the complex factors, particularly those experienced by the college population, that influence consent communication as well as highlights the complicated interplay between factors at all levels of the model. 


\section{Summary of Findings}

This study explored a variety of individual, interpersonal, community, and societal predictors of consent communication. Hypothesis and research questions addressed variables such as empathy, perceived behavioral control, metacommunication, and ongoing negotiation. Findings indicate that there are a variety of factors that influence college students' consent communication. Results shared below have numerous implications for communication scholars as well as higher education professionals and college health practitioners who work to address consent at colleges and universities.

\section{Consent at the Individual Level}

Perceived behavioral control. Perceived behavioral control is an important component to understanding human behavior. The present study examined perceived behavioral control in a variety of ways in order to examine its effect on consent communication. First, no statistically significant difference in perceived behavioral control was found between students who reported receiving prior consent education and students who did not receive consent education. Perceived behavioral control was also examined in relation to perceived level of preparation to navigate sexual situations. Results indicated that while the level of preparation did predict perceived behavioral control, students' level of preparation only accounted for a small amount of variance in perceived behavioral control.

These findings are peculiar because, intuitively speaking, prior education on a topic should increase one's feelings that they can perform behaviors specific to that area. Logic would also follow that preparedness would also increase confidence to perform behaviors. Humphreys and Brousseau (2010) explained that key behavior control issues in the context of sexual consent are ideas of reduced pleasure, feeling awkward, and confidence level to negotiate consent. 
Perhaps in consent situations, these behavioral factors overrule or at least are more important than information received in educational settings. However, due to participants indicating that school is the third most influential source of consent information, this may not be true.

Another explanation for prior consent education's lack of effect on perceived behavior control is that perhaps educational initiatives are not addressing the right issues. The majority of U.S. high schools provide abstinence-only education that tend to not be inclusive, often include inaccurate information, and do not address consent (Kantor et al., 2008; Muehlenhard et al., 2016). While many institutions of higher education have implemented facilitated lectures, workshops, and educational campaigns to increase awareness of sexual assault and consent, none have demonstrated outcomes specific to consent (Beres, 2014). In addition, many of these programs do not reflect the sexual scripts of college students and do not clearly define what constitutes consent (Johnson \& Hoover, 2015; Muehlenhard et al., 2016; Reynolds, 2004). If programs addressing consent are not connecting with students or reflecting their lived experience, it makes sense that they would not have a positive effect on students' thoughts and behaviors.

Empathy. Empathy is an important component of interpersonal communication. While previous research has linked empathy to aggression, no studies exist that examine both empathy and consent. The present study identified important links between empathy and consent communication. Results indicated that as empathy increased, both favorable attitudes toward establishing consent and consent norms increased. An increase in perceived behavioral control was also associated with higher empathy scores. Collectively, these results demonstrate that increased student empathy should positively influence college student beliefs and actions surrounding sexual consent. Additionally, empathy positively correlated with nearly all scale 
variables at a statistically significant level, including perceived behavioral control, attitudes towards establishing consent, approaches to establishing consent, consent norms, and metacommunication.

These findings concur with what is known about empathy as it relates to interpersonal communication. Since empathy helps individuals understand and assess others' mental and emotional states in order to inform their own behavior, it makes sense that empathy would play an important role in consent communication (Bruneau, 2009; Eisenberg, 2000). This also may explain some of the disconnect among current young adults actively communicating consent. Today's college students score $40 \%$ lower in empathy than students a decade ago. Lack of interpersonal skills, due in part to the rise of electronic communication, is theorized to be part of this decline (Dolby, 2014; Konrath et al., 2011). It is possible that these communication issues could spill over into sexual situations. If students are not used to face-to-face communication in other contexts, then they are not likely to use these skills in intimate situations either.

The findings of this study are not surprising, given what is known about empathy's effects on communication. Because empathy involves understanding what others are thinking and feeling in order to inform one's behaviors (Bruneau, 2009; Eisenberg, 2000), it makes sense that empathy would affect consent attitudes and behaviors. Even though sexual situations are intimate and private, they are also social in that social construction helps form how people respond in sexual situations.

More concerning, results indicate that as empathy decreases college students may be more likely to have unfavorable feelings towards establishing consent and accept negative consent norms. Additionally, a decrease in empathy may lead individuals to feel that they are less able to perform consent communication. These findings are extremely troublesome given 
what we know about today's college students' empathetic capacity. Individuals who score higher in empathy show more emotional sensitivity and self-control, which in turn effect their information processing and decision making (Konrath et al., 2011; Olderbak et al., 2014; So et al., 2015). These findings highlight empathy's important role in ensuring sexual situations are successfully negotiated.

College student preparation. Existing research illustrates that for a variety of reasons, today's students are not prepared to navigate sexual situations experienced at college (Muehlenhard et al., 2016). Self-reported scores for participants in the present study directly contradict this information. Interestingly, $82 \%$ of participants indicated that someone had directly discussed sexual consent with them. Of those who indicated someone had spoken to them about consent, nearly $68 \%$ indicated they received information from school/sex education.

Additionally, when giving themselves a percentage grade for preparation participants on average gave themselves a grade of B for feeling adequately prepared to navigate sexual situations (86\%) and a grade of A for feeling adequately prepared to talk about consent with sexual partner(s) $(92 \%)$. It is important to point out that less than $13 \%$ of participants were underclassman, with the majority identifying as fourth year or graduate students. It is possible that younger students may be less confident in their abilities; however, data collected in this study cannot answer that question due to low participation of first and second year students. In fact, low participation of first and second year students may indicate their uneasiness with the topic of sexual consent.

Student level of preparation was identified as a predictor of perceived behavioral control. Given what is known about perceived behavioral control, it is logical that self-reported higher levels of preparation would indicate feeling better prepared to take action. However, the self- 
reported component is important to note. By design, prior consent education and preparation was used broadly and largely defined by the participant. Additionally, students may overestimate their preparedness to navigate sexual situations and talk about consent with their parents. While self-report measures are always at risk to be influenced by self-report bias, the factors measured by this study may in particular be influenced by social desirability.

Social desirability is a response bias that can lead to participants to answer questions based on factors outside of the question content and in turn attributing socially desirable attitudes and behaviors to themselves (Johnson \& van de Vijver, 2002; Ones, Viswesvaran, \& Reiss, 1996). Since conceptually consent is widely accepted as a desirable behavior, students may have responded to the survey in a manner that inflates their tendencies towards consent. However, researchers note that for the most part social desirability's effect on participant responses is minimal and does not typically overtly alter findings (Ones et al., 1996; Visschers, Jaspaert, Vervaeke, 2017).

Additionally, prepared to talk about consent with sexual partner was a stronger unique predictor of perceived behavioral control than participant's feelings of being able to navigate sexual situations as a whole. This finding highlights the importance of communication in consent situations because if students do not feel they can talk about consent with their current partner, they may not feel overall prepared to navigate sexual situation. This also indicates a potential relationship between consent communication skills and behavioral intent.

Year in school. While participant year in school did not have a statistically significant difference on any scale variables assessed, there was a visible upward trend observed in the variable means for all scales by year in school. These results indicate that as year in school increased, student favorable attitudes and consent supportive behaviors increased. This may 
indicate that year in school may influence consent communication; however, this trend could also be an artifact of this specific sample of students. Additional research with a more varied student sample needs to be conducted in order to further examine this phenomenon.

\section{Interpersonal Aspects of Consent}

Sexual and gender minorities. The majority of existing consent and sex script research focuses on heterosexual males and females, meaning that little is known about the consent behaviors of gender and sexual minorities. For this reason, the present study sought to determine if there were any differences between students with varying sexual orientations and gender identities. The findings did not indicated statistically significant differences in attitudes toward consent, approaches to establishing consent, and consent norms after controlling for sexual orientation. Additionally, no statistically significant difference was found in attitudes toward establishing consent, approaches to establishing consent, and consent norms after controlling for gender identity.

Notably, despite targeted outreach to try to include diversity in sexual orientation and gender identity, very few participants identified as a sexual or gender minority. Only $19 \%$ of participants identified as non-heterosexual and less than $2 \%$ identified as gender nonconforming. Of those that identified as agender or fluid/genderqueer, slight differences in means were observed. This could indicate that gender identity may be worth exploring in future research with an increased participant pool of gender non-conforming individuals.

While the results of this study could indicate that no differences exist between nonheterosexual and gender non-conforming students and their peers, these findings could also reflect the small numbers of gender and sexual minorities that participated in the study. Because individuals within the LGBTQ+ community are at higher risk for sexual assault and less likely to 
report assaults, it is important to understand unique characteristics of individuals in these communities (Long et al., 2007; White House Council on Women and Girls, 2014). Since very little research exists that examines the sexual and consent communication behaviors of nonheterosexual and gender non-conforming individuals, more research needs to be done in these areas in order to fully understand the sexual behaviors of all college students.

Additionally, very little variance was observed in mean scores for all variables between males and females. This contradicts Jozkowski's (2011) findings that found significant difference in how males and females communicate consent. Jozkowski's results aligned with traditional sex scripts where men are expected to initiate sex and women are expected to serve as sexual gatekeepers who decide whether a sexual encounter will occur. It is important to note that Jozkowski's research was conducted with heterosexual males and females.

Consent as an ongoing process. Ideally, consent should be continuous throughout and beyond the sexual encounter (Reynolds, 2004). However, the focus on "yes means yes" may give the impression that consent is a one-time agreement at the beginning of a sexual encounter, regardless of relationship type. Metacommunication, or partner communication about how the relational partners communicate, is an important component of a relationship that is tied to the well-being and feelings of equity of those in the relationship (Knoblach et al., 2006). Since consent communication is largely tied to equity in sexual situations, the present study explored whether discussion of sexual activity and consent outside of sexual encounters affected whether or not consent was viewed as an ongoing process.

Results tied to metacommunication and ongoing negotiation are interesting. While metacommunication was found to be a statistically significant predictor of consent as an ongoing process, metacommunication only accounted for a very small amount of change in ongoing 
process. The small amount of variance in ongoing process caused by metacommunication could be due to the fact that when relational metacommunication takes place, it is rarely about intimate physical activity (Baxter \& Wilmot, 1984).

Also noteworthy is that ongoing negotiation positively correlated at a statistically significant level with all scale variables except approaches to establishing consent and empathy. Results of correlation tests indicated no relationship between ongoing negotiation and empathy. Ongoing negotiation showed a statistically significant, negative relationship with approaches to establishing consent. While these results could be an anomaly of this study's sample, these findings are interesting. These results could also reflect college student's ambivalence to communicate verbally in sexual situations (Humphreys, 2004; Humphreys \& Brousseau, 2010; Johnson \& Hoover, 2015; Jozkowski et al., 2014; Lim \& Roloff, 1999). If this is the case, the cause needs to be explored to determine why students will not communicate verbally in intimate situations.

It is important to note that after exploratory factor analysis, the ongoing negotiation scale only included two items. While the validity and reliability results for these two items were strong, this is a less than desirable number of items for a scale. Despite this, these results indicate that ongoing negotiation may very well be an important part of consent communication and this aspect of consent communication needs to be explored further.

\section{Community Impacts on Consent}

Prior consent education. According to prior research, college students are not prepared for the sexual situations they are exposed to at college - partly because of a lack of comprehensive sex education in high schools that discuss consent (Kantor et al., 2008;

Muehlenhard et al., 2016). However, self-reported scores for participants in this study directly 
contradict this information. More than $82 \%$ of participants indicated that someone had spoken with them directly about consent. Of those who indicated someone spoke with them about consent, $68 \%$ indicated they received information from school/sex education. These high percentages could be an artifact of that participants were largely fourth year and graduate students. These scores could also be due to the fact that the institution this study was implemented at has conducted an online educational program focused on consent for the past three years. Although there is no way to know how many participants were returning students, as opposed to transfer students, it is possible that this educational program contributed to these high numbers.

Findings also showed that prior consent education had no statistically significant effect on the individual factors of attitudes toward establishing consent, approaches to establishing consent, and consent norms. Means for these variables were also relatively similar. These findings are curious because one would think that prior exposure to educational messaging in this area would in turn effect these variables. Another odd finding is that higher mean scores were observed for those who did not receive prior consent education in the areas of attitudes toward consent and consent norms. These findings could be due in part that most sex education delivered in high schools focuses on abstinence (Muehlenhard et al., 2016). Abstinence-only programs do not in any way address consent, which could account for this variance in mean scores. Also, by design the term "prior consent education" is left up to the participant to define. It is possible that participants confused sex education for consent education. Another explanation for this finding could be that whatever education participants received was not effective or did not address these specific areas. However, participants did list school as the second most influential source of information on consent. This incongruence may be worth exploring more in 
future research, as well as investigating in more depth the types of school-based sexual education and when students received it.

Alcohol and college. While not a main focus of the present study, alcohol and sex are inextricably linked with each other in the context of the college experience (Ward et al., 2012). Because alcohol is a large part of college students' sexual experiences, information was collected regarding how often participants engage in sexual activity under the influence of alcohol. Results indicated that $44 \%$ of participants reported engaging in sexual activity while under the influence of alcohol some or most of the time. Moreover, the present study found a weak positive correlation between participant consent norms and frequency of engaging in sexual activity while under the influence. A weak, positive relationship was also found between approaches to establishing consent and frequency of engaging in sexual activity under the influence. These findings indicate that the more frequently students participated in sexual activity while under the influence of alcohol, the more clear they believed they communicated and understood consent signals.

These findings have disturbing implications for communication in consensual sexual situations. First, prior research establishes that young adults' usage of indirect and nonverbal methods to communicate consent put even sober, well intentioned people at risk for inaccurately interpreting their partner's consent signals (Hall, 1998; Hickman \& Muehlenhard, 1999; Johnson \& Hoover, 2015; Jozkowski, 2011). Previous research also indicates that miscommunication about sexual consent may contribute to sexual aggression (Jozkowski et al., 2014). If students lack the abilities and interpersonal skills to accurately assess consent communication in an unaltered state, alcohol will likely exacerbate the problem. 
These results are shared with caution, because the top rape myth accepted by college students is that sexual assaults happened due to miscommunication (Vandiver \& Rager Dupalo, 2012). It is vital to note that alcohol use alone does not cause sexual assaults, perpetrators do. However, research indicates that predators sometimes use alcohol as a weapon (Lake, 2015). The weaponization of alcohol includes camouflaging the amount of alcohol someone is ingesting with sweet drinks and looking for vulnerable individuals who have ingested large amounts of alcohol who may be easy to take advantage of either because of incapacitation or because they are easy to isolate from their friends (Lake, 2015). However, to reiterate, alcohol does not cause someone to be assaulted. Instead, perpetrators use alcohol as a tool to make someone an easier target for an assault. Additionally, when sex and alcohol are mixed, acts meeting the legal definition of rape are more likely to occur (Task Force of the National Advisory Council on Alcohol and Alcoholism, 2002).

Setting aside alcohol's complicated relationship with sexual assault, alcohol also greatly alters the dynamics of consensual sexual situations (Muehlenhard et al., 2016). Some students particularly males - may suffer from alcohol myopia, where the most immediate and salient cues in a situation might be noticed, but subtle cues and long-term consequences are more likely to be missed (Steele \& Josephs, 1990). Alcohol also changes the dynamics of sex scripts. Men perceive more sexual intent in women than sober men, paying more attention to cues of interest and less attention to ambiguous cues or those that show disinterest (Abbey, Zawacki, \& Buck, 2005). What is not clear is why college students often engage in sexual activity while under the influence. Drinking is sometimes used as a social lubrication, so it is possible that college students use alcohol to loosen up in sexual situations due to feeling unsure or unprepared. 
Often, higher education is reluctant to talk about student alcohol use - particularly when it comes to sex. However, students must understand alcohol's effect on their abilities to negotiate consent and the legal ramifications of engaging in sexual activity under the influence as well as predatory uses of alcohol. In addition, focusing on building interpersonal communication skills such as empathy may equip students with a strong enough foundation to help them successfully navigate consent while under the influence while ensuring to explain inability to consent when incapacitated.

\section{Societal Influences on Consent}

College students are exposed to messages about sex and consent from a variety of sources. It is important for professionals to be cognizant of student sources of influence in order to counter their influence, if necessary. In order to assess the level of influence that a variety of consent information sources, students ranked sources one to seven with one being. Friends were by far the most influential factor, followed by school, parents/guardians, and media. However, there was only a .21 difference in mean scores of school, parents/guardians, and media. Participants reported religion as the least influential. The present study's findings that parents/guardians extends on prior research that shows parental influence on college students decisions on behaviors such as alcohol and substance use (Carpenter, 2009). The present study's findings also somewhat support previous research that found that media sources were of greater influence on sexual intentions than school and religion (L'Engle et al., 2006).

The socially constructed nature of consent makes outside forces, such as friends and media, important factors in informing consent attitudes and behaviors. Participants rating media sources as influential is highly problematic due to how sex and consent are often depicted. Many view our current society as a rape supportive culture, which is the exact opposite of a consent 
culture. These types of environments normalize sexual aggression (Herman, 1984). Rape culture serves as a link that connects gender, socialization, media, and institutions, ultimately modeling anti-consent behaviors (Guckenheimer, 2008). Aggression in sexual situations not only becomes normal, it becomes sexy.

Media sources bombard individuals with messages that suggest communication, negotiation, and equality are unnecessary or impossible in sexual encounters which leads them to adopt as the truth (Hust et al., 2014; Reinholtz et al., 1995; Werder, 2009). These sources reinforce that consent is not part of the traditional sex script and exposure to these messages are negatively associated with consent negotiation intentions (Hickman \& Muehlenhard, 1999; Hust et al., 2014). In addition, it is likely that many students receive information from similar media sources meaning that media influence likely also impacts students' top reported influencing source: their friends.

Students may have listed religion as least influential for a variety of reasons. First, this study targeted sexual active individuals and nearly all participants were currently or had at some point been sexually active. Due to the beliefs of some religions, this may have excluded certain individuals who choose to not be sexually active prior to marriage. Also, participants were not explicitly asked if they were married. Due to the fact that the majority of participants were fourth year and graduate students, it is conceivable that some of them may be married. For this reason, religion may have less of an impact on consent. For younger students in particular, college tends to be a time of freedom and exploration which may outweigh religious preferences. Additionally, religious affiliation was not measured so it is unknown how many participants affiliate with a certain belief system. 


\section{Implications}

The present study highlights important areas of contribution for the field of communication as well as numerous practical implications for professionals working with sexual consent initiatives. With the majority of prior consent research approaching the topic in the context of sexual assault, communication scholarship can provide practitioners with a better understanding of the factors that influence consent communication at the individual, interpersonal, community, and societal levels. Addressing aspects at all levels of the socialecological model ensures a holistic approach that can lead to true personal and societal change. Consent efforts on college campuses can address the communicative phenomena in a variety of ways, including defining consent as a verbal communication, building interpersonal skills that improve students' confidence and abilities to communicate in sexual situations, addressing sources of influence, and incorporating alcohol awareness into interventions.

\section{The Role of Communication}

Overall, the findings of this study emphasize the centrality of communication to navigating sexual consent situations. Because the majority of research studies on sexual consent have taken place in the fields of psychology and sociology, communication scholars can provide unique insights into consent norms and behaviors that impact sexual encounters. By using communication theories and concepts to further investigate consent, communication scholars can contribute productively to existing research in this area as well as help create better informed interventions to help improve communication skills necessary to have mutually enjoyable and equitable sexual situations.

Communication is an important part of any type of relationship, and is closely tied to the vitality of the relationship, feelings of equity, and the overall well-being of the individuals in the 
relationship (Knobloch et al., 2006; Miller-Ott \& Linder, 2014). Because communication defines relationships and implies commitment, the field of communication can also contribute greatly to understanding metacommunication and ongoing negotiation about consent (Watzlawick et al., 1967). Knowledge about consent negotiation must be situated within a broader understanding of behaviors and experiences. The field of communication can help establish if findings from this study are indicative of larger patterns that have broader communicative implications.

Additional research in the areas of metacommunication and ongoing negotiation will also help practitioners know how to move beyond "yes means yes." While the shift from "no means no" to "yes means yes" sought to clarify consent situations by instructing partners to verbally grant and obtain affirmative consent, this focus makes consent seem like a one-time agreement (Reynolds, 2004). In reality, consent should be obtained for all sexual activities in a sexual encounter and for every sexual encounter - no matter the relationship between partners. To better inform messaging and interventions addressing ongoing negotiation and encourage partners to discuss intimate acts, more research needs to be done to understand students' communicative patterns as well as what intervention tactics will be effective.

Additionally, consent must be defined and explained as being a communicative act. Unless there are clear verbal signals, ambiguity in consent situations will persist (Lim \& Roloff, 1999). Communication scholars and/or professionals should work alongside higher education professionals in a variety of ways that highlight consent as a communicative act. Areas for collaboration include working together to help ensure policies are written in a manner that clearly highlights the communicative threshold for consent, creation of education and campaign messaging, and assessment of the effectiveness of interventions. 


\section{Interpersonal Skill Building}

The findings of this study illustrate the need to shift educational and campaign focuses toward building interpersonal communication skills in order to help students more effectively communicate consent. Currently, consent on college campuses is typically approached as a way to prevent sexual assaults (Borges, Baynard, \& Moynihan, 2008). Some initiatives have shown positive outcomes in areas such as increased awareness of rape myths, empathy for victims, bystander behavior, and overall awareness of sexual assault (Bradley et al., 2009; Foubert et al., 2010; Hanson \& Gidycz, 1993; Johnson \& Hoover, 2015; Jozkowski \& Peterson, 2014; Reynolds, 2004). However, many programs and efforts do not clearly define what constitutes consent and very few incorporate aspects of the typical college sex script. Higher education's focus needs to shift from viewing consent from a necessary threshold to avoid committing a crime to one that highlights consent as a vital component of an equally enjoyable sexual encounter and an overall vital part of a healthy relationship.

While consent as a component of healthy relationships does not need to be mutually exclusive from sexual assault prevention effects, college professionals should consider approaching consent in a manner that emphasizes building skills necessary for healthy relationships without a focus on crime mitigation. Shifting the focus to improving consent supporting behaviors is reinforced by Borges et al.'s (2008) finding that students showed greater knowledge gain when they engaged in discussion and participated in an activity. The present study, combined with Borges et al.'s (2008) findings, also highlights the importance of focusing on positive and healthy behaviors versus crime-focused or scare tactics. Consent is fundamental to how individuals make decisions about and negotiate healthy sexual relationships (Borges et al., 2008). 
Increasing interpersonal skills that help individuals understand and assess another's thoughts and feelings, as well as building the skills necessary to talk about their appraisals, will greatly clarify and improve consent communication. The findings of the present study combined with previous empathy research illustrate the vital role of empathy in consent situations. Because college students' capacity for empathy has declined over the past decade (Konrath et al., 2011), this is an important area for practitioners to consider. Understanding empathy and the causes leading to the decrease in empathy of today's college students can help mitigate these negative effects and improve empathy (Konrath et al., 2011). Ways to foster empathy include increasing emotional awareness of self and others, improving interpersonal communication skills, decreasing reliance on technology, and using media literacy to help cultivate awareness of technology use on empathetic skills.

Additionally, empathy's role in consent also highlights the need for work addressing elements of consent to begin earlier than college. Prior research shows that empathy is a teachable skill to both children and young adults (Feshbach \& Cohen, 1998; Hatcher et al., 1994). Elementary school-based programs that help build empathy have been shown to decrease aggressive behavior and increase prosocial behaviors (Konrath et al., 2011). Teaching these skills early is a much more proactive approach than addressing them in college. Furthermore, working to build a foundation of respect in children may help also lead to an overall more consent supportive culture.

In regard to metacommunication, students need to know that if they intend to engage in intimate activity that they need to talk to each other about activities which are about to take place. Communication defines even the most casual of relationships (Watzlawick et al., 1967). Even when metacommunication takes place in a relationship, it is rarely about physical aspects 
of the relationship (Reherman, 1987). Since the ability to metacommunicate is an important component of successful communication and enhances individuals' awareness of others, it is central to skill building necessary to improve consent communication (Watzlawick et al., 1967). Students must have the confidence and skill necessary to be able to communicate with their sexual partners. This will not only help with the successfully negotiation of consent, it will also help increase their well-being and feelings of equity in the relationship (Knobloch et al., 2006; Miller-Ott \& Linder, 2014).

Sexual consent interventions should also focus on the ongoing nature of consent communication. While the shift from "no means no" to "yes means yes" sought to clarify consent situations by instructing partners to say and obtain affirmative consent, it can also be misleading and make consent seem like it a one-time agreement (Reynolds, 2004). Additionally, the ongoing nature of consent negotiation is not reflected in media portrayals and established sex scripts (Hickman \& Muehlenhard, 1999; Reynolds, 2004). In order to counter these influences, consent initiatives must teach students to continually check in with one another for each sexual activity and for every sexual encounter. Students may need to be given realistic examples that explicitly illustrate what ongoing negotiation may look like in sexual situations. Furthermore, incorporating verbal communication techniques such as face-saving and humor may prove effective in helping college students navigate sexual communication in a way that makes the situation less awkward (Miller-Ott \& Linder, 2014).

Additionally, findings of the present study indicate that interventions may need to vary depending on students' year in school. Since results indicated that perceived skill level tends to increase as year in school increases, interventions should adapt to reflect this as well as to acknowledge the variety of consent situations that college students experience. Often higher 
education professionals tend to focus on the hook up culture due to the risks that accompany this type of sexual activity. However, consent in the context of a relationship is just as important and should not be ignored.

\section{Addressing Community and Societal Influences}

While it is important to examine consent at the individuals and interpersonal levels, the outer levels of the social-ecological model are important to attend to the entire scope of consent influence. Addressing consent at all levels is necessary in order to transform the current rapesupportive culture into one that values and affirms consent. Influences at the community and societal levels greatly affect an individual's thoughts, norms, and actions that influence interpersonal communication. Community factors such as alcohol use and college policies as well as broader societal influences are addressed below.

Higher education professionals cannot be hesitant to talk about sex and alcohol with students. Findings from the present study supports existing research which shows, that for some students, sexual encounters and alcohol often go hand-in-hand (Ward et al., 2012). Alcohol must be included in consent education because, for some students, alcohol is inextricably linked to the college sexual experience. Typically, most schools emphasize the fact that legally individuals cannot consent when incapacitated by drugs or alcohol, which is very important for students to understand. However, students will continue to have sex under the influence. In addition to discussing the legal implications of consent under the influence, students need to understand how alcohol affects their ability to communicate and understand someone else's communicative signals. Furthermore, improving sober communication skills should positively enhance consent communication when under the influence. 
Institutions of higher education must continue to work toward defining consent in a manner that requires affirmative, verbal communication and clearly explains what that means for communicative behavior. Student lack of preparedness for college sexual situations, combined with societal influences that directly contradict verbal consent, may lead students to rely on indistinguishable, nonverbal methods of obtaining consent. While university policies are intended to reduce instances of sexual assault, ambiguous consent policies leave students unsure of what these guidelines mean for actual behavior (Borges et al., 2008). Affirmative, verbal communication should be clearly stated and explained in policies. While this goes against the current sexual scripts and preferences of college students, research also tells us that new sex scripts are able to emerge through social construction (Laumann \& Gagnon, 1995; Masters et al., 2013). This is just one way that higher education institutions can help facilitate the transformation from a rape-supportive society to one that normalizes consent.

Practitioners working to address consent on college campuses must also be aware of consent sources of influence. Results from the present study indicate that the most influential sources of consent information are students' friends, parents/guardians, school, and media. Future interventions must consider these sources, the messages students acquire from these sources, and how to use or counter information received. As a whole, the majority of messages that college students receive, particularly from the media, are not supportive of consent (Hust et al., 2013; Sprecher et al., 1994). Of particular concern are rape myths that college students endorse, such as sexual assaults occur due to miscommunication (Derning et al., 2013; Koss et al., 1988; Vandiver \& Rager Dupalo, 2012). Because student sources of influences affect them in different ways (Hust \& Brown, 2008; L’Engle et al., 2006), initiatives should address information sources separately in order for students to be able to critically assess information 
they receive. Additionally, parental influence may be an area for higher education to capitalize on. Sharing information with parents would help another area of students' lives reinforce consent-supportive messaging as well as help normalize consent.

Lastly, higher education should use consent education, campaigns, and interventions to help counter media messages students are exposed to that contribute to the current rapesupportive culture. The majority of sexualized media content does not include consent communication, normalizes aggression, and can lead to sexual violence (Boux \& Daum, 2015; Guckenheimer, 2008; Herman, 1984; Werder, 2009). Improving media literacy may help students analyze media messages they receive in order to be able to identify these sexual situations as not realistic in order to help counter the messages' negative effects. Higher education can also try to work with the media to change their portrayals of sexual situations. Much like public health lobbying has reduced media representations of smoking, college health professionals can appeal to media sources to build consent into depictions of sexual situations.

When examining the societal influences that lead to rape-supportive cultures, expecting higher education to end sexual assault experienced by college students is not a proactive approach and is very much a Band-Aid fix that does not address root causes. Colleges and universities cannot be the only sources of consent information. All areas of an individual's life need to work together to normalize consent and help build skills necessary to successfully navigate sexual situations. This multi-pronged approach that addresses individual and interpersonal communication skills as well as community and societal influences is more likely to achieve success. 


\section{Study Limitations}

The present study has several limitations worth noting, including survey and measurement considerations as well as factors that affect generalizability. Initially, ambiguity in key survey terms could have altered how participants responded. Participants themselves were intentionally allowed to define key terms such as sexual activity, sex education, and even sexual consent. It is likely that individuals approach these terms in different ways. Varying definitions of sex education are particularly worth noting since the majority of participants responded that they had received sex education; a pattern which is contrary to national data.

Factor analysis uncovered measurement issues with a few of the survey scales. In some instances, the factor analysis completed for this study did not reconcile with what previous researchers had found. For instance, ongoing negotiation scale only had two items remaining in order to get the scale to load on one factor and these items were questions created specifically for this study. Further development work with ongoing negotiation will help ensure that the scale is measuring what researchers intend as well as clarify if this construct needs to be measured in multiple scales. Other scales, even though they were based on previously used measurements, did not replicate previous studies' factor analysis results. While these could be anomalies of this specific data set, additional scale development work is recommended in order to confirm these measures are the best tools for examining sexual consent communication.

Additionally, the age and year in school of participants in this sample skewed older than anticipated. More than half of respondents were fourth year or graduate students, and only one first year student completed the survey. While upperclassman and graduate students are important groups to gather data from, an equal breakdown of all school years would have provided data more representative of the entire college experience. This predominantly older 
sample hinders the ability to generalize results for all college students; however, it also provides an opportunity for future research to compare this study's results to first and second year students. Furthermore, while no significant findings were found among scales based on participant year in school, there were slight increases in participant scale scores as their time in school increased. This also illustrates that participant year in school is worth exploring in future research.

A few other aspects may impact this study's generalizability. Ultimately, a truly random sample was not achieved. While some participants were recruited through random sampling procedures, additional methods were used partially by design and partially due to poor response rates. Snowball sampling was intentionally used in order to actively recruit participation from individuals in the LGBTQ+ community. Convenience sampling was employed due to poor response rate to initial random sample recruitment efforts. Furthermore, institutional factors, such as the fact that all returning students receive an online educational course addressing consent, reduce the ability to make generalizations based on this study's findings.

It is also important to note that this study focused on students who were currently sexually active or had been in the past. While this was an intentional decision, the attitudes, beliefs, communication patterns, and preparation levels of non-sexually active students are important to explore as well. Future research could expand upon existing research by targeting abstinent as well as newly sexual active college students to their investigate predictors of consent communication.

Lastly, participants could have skewed their answers due to self-report bias. Since previous research indicates that college students are not prepared for college sexual situations, results from the present study may indicate that college students overestimate their consent 
abilities. Additionally, because of the sensitive nature of sexual consent, social desirability bias may add another layer of complexity to this study's findings. Triangulation with additional research methods other than closed-question survey would also help strengthen findings from this study as well as others that have explored consent. For instance, qualitative data from focus groups or in-depth interviews, could enrich our understanding of consent communication.

\section{Opportunities for Future Research}

An important take-away from this study is that there are many opportunities for future consent communication research. The present study uncovered interesting links among elements of consent communication and empathy. Just as consent needs to be examined and discussed outside of the context of sexual assault, empathy needs to be explored outside of sexual aggression. Future research should build upon this study's results to further investigate empathy in regard to building interpersonal skills that aid students in consent situations as well as identifying additional implications for college students and higher education.

Future research should also explore ongoing negotiation in more depth. Because consent is an ongoing process that continues throughout the sexual encounter, it is imperative that young adults understand this aspect of consent communication. While the ongoing negotiation scale for the present study included a less than desirable number of items for a complete scale, the variable still yielded interesting and worthwhile findings. Future research should explore this variable and work to create a more complete scale to measure this phenomenon.

Additional research should continue to investigate diversity of participants as well as gathering data across multiple institutions. It is vital that future research includes representation from all sexual orientations, gender identities, race/ethnicities, and ages, so that data generated and interventions created are representative of the entire college population. Since the present 
study had so few first and second year students, additional research should also include younger students as well as incoming students to serve as a comparison to this study's data. Exploring consent across multiple institutions of higher education would help mitigate any institutional factors as well as greatly increase the generalizability of findings.

Lastly, future researchers exploring consent should consider utilizing experimental research designs. The vast majority of existing research, including the present study, have been close-ended surveys. Triangulation with additional methods will strengthen existing scholarship while also providing unique new insights into beliefs and behaviors. Experimental research designs, such as manipulations that take participants through role playing of consent situations and include a pre and post-tests to assess learning outcomes, would complement the existing baseline data. Another important next step for research in this area is to test consent interventions. Practitioners should move beyond program evaluations to conduct formative evaluation with students to help ensure that interventions resonate with their intended populations prior to implementation.

\section{Conclusion}

The majority of existing research on sexual consent has been conducted in the realms of sociology and psychology, often focusing on consent in the context of rape and sexual assault. The field of communication has much to offer in regard to researching consent, because, after all, consent is a communicative act. The present study's findings add to the growing amount of literature that has the same goal in mind: mutually enjoyable sexual encounters where consent is freely and equally given. The findings of the present study indicate that individual factors that affect interpersonal communication such as empathy and perceived behavioral control are important indicators of consent norms and communication. 
In our hyper-sexualized age, a true culture shift is needed in order to transform from the current rape-supportive culture to a consent normative culture. Examining and attending to factors that affect consent at all levels of the social-ecological model is an approach that is more likely to garner sustainable personal and societal change. By addressing a broad spectrum of consent communication predictors, a true culture shift can take place in order to empower people to have autonomy over their own bodies and respect for other through communication skills necessary to successfully navigate sexual situations. 


\section{REFERENCES}

Abbey, A., Zawacki, T., Buck, P. O. (2005). The effects of past sexual assault perpetration and alcohol consumption on men's reactions to mixed signals. Journal of Social and Clinical Psychology, 25, 129-155. doi: 10.1521/jscp.24.2.129.62273

Abbey, A., Zawacki, T., Buck, P. O., Clinton, A. M., \& McAuslan, P. (2016). Alcohol and sexual assault. Washington, DC: National Institute on Alcohol Abuse and Alcoholism. Retrieved from: https://pubs.niaaa.nih.gov/publications/arh25-1/43-51.htm

Ajzen, I. (1991). The theory of planned behavior. Organizational Behavior and Human Decision Processes, 50, 179-211. doi: 10.1016/0749-5978(91)90020-T

Ajzen, I. (2002). Perceived behavioral control, self-efficacy, locus of control, and the theory of planned behavior. Journal of Applied Psychology, 32, 665-683. doi: 10.1111/j.15591816.2002.tb00236.x

Armitage, C. J., Conner, M., Loach, J., \& Willetts, D. (1999). Different perceptions of control: Applying an extended theory of planned behavior to legal and illegal drug use. Basic and Applied Social Psychology, 21, 301-316. doi: 10.1207/15324839951036326

Baxter, L., \& Wilmot, W. (1984). Secret tests: Social strategies for acquiring information about the state of the relationship. Human Communication Research, 11, 171-201. doi: 10.1111/j.1468-2958.1984.tb00044.x

Beres, M. A. (2007). "Spontaneous” sexual consent: An analysis of sexual consent literature. Feminism \& Psychology, 17, 93-108. doi: 10.1177/095935307072914

Beres, M. A. (2014). Rethinking the concept of consent for anti-sexual violence activism and education. Feminism \& Psychology, 24, 373-389. doi: 10.1177/0959353514539652 
Beres, M. A., Herold, E., \& Maitland, S. B. (2004). Same-sex sexual consent scale. Archive of Sexual Behavior, 33, 475-486. doi: 10.1023/B:ASEB.0000037428.41757.10

Boone, T. L., \& Lefkowitz, E. S. (2004). Safer sex and the health belief model: Considering the contributions of peer norms and socialization factors. Journal of Psychology \& Human Sexuality, 16, 51-68. doi: 10.1300/J056v16n01`04

Borges, A. M., Banyard, V. L., \& Moynihan, M. M. (2008). Clarifying consent: Primary prevention of sexual assault on a college campus. Journal of Prevention \& Intervention in the Community, 36, 75-88. doi: 10.1080/10852350802022324

Boux, H. J., \& Daum, C. W. (2015). At the intersection of social media and rape culture: How Facebook postings, texting and other personal communication challenge the "real" rape myth in the criminal justice system. Journal of Law, Technology, and Policy, 1, 148-186. Retrieved from: http://www.heinonline.org/HOL/Page?handle=hein.journals/ jltp2015\&div=7\&collection=journals

Bradley, A., Yeater, E., \& O’Donohue, W. (2009). An evaluation of a mixed-gender sexual assault prevention program. Journal of Primary Prevention, 30, 697-715. doi: $10.1007 / \mathrm{s} 10935-9-0198-4$

Bruneau, T. (2009). Empathy. In S. W. Littlejohn \& K. A. Foss (Eds.). Encyclopedia of communication theory (pp. 337-340). Thousand Oaks, CA: Sage.

Buchwald, E., Fletcher, P., \& Roth, M. (1993). Transforming a rape culture. Minneapolis, MN: Milkweed Editions. 
Burnett, A., Mattern, J. L., Herakova, L. L., Kahl, Jr., D. H., Tobola, C., \& Bornsen, S. E. (2009). Communicating/muting date rape: A co-cultural theoretical analysis of communication factors related to rape culture on a college campus. Journal of Applied Communication Research, 37, 465-485. doi: 10.1080/00909880903233150

Buunk, B. P., van den Eijnden, R. J. J. M., \& Siero, F. W. (2002). The double-edged sword of providing information about the prevalence of safer sex. Journal of Applied Social Psychology, 32, 684-699. doi: 10.1111/j.1559-1816.2002.tb00237.x

Carpenter, C. M. (2009). Development of a Drug Use Resistance Self-Efficacy (DURSE) Scale. American Journal of Health Behaviors, 33, 147-157. doi: 20.5993/ajhb.33.2.4f

Centers for Disease Control and Prevention. (2015, March 25). The social-ecological mode: A framework for prevention. Retrieved from https://www.cdc.gov/violenceprevention/overview/social-ecologicalmodel.html

Cline, T. R. (1979). A Markov analysis of strangers, roommates, and married couples conversational focus on their relationships. The Southern Speech Communication Journal, 45, 55-68. doi: 10.1080/10417947909372437

Corteen, K. (2004). Beyond (hetero)sexual consent. In M. Cowling \& P. Reynolds (Eds.). Making sense of sexual consent (pp. 171-195). Burlington, VT: Ashgate.

Cowling, M., \& Reynolds, P. (2004). Introduction. In M. Cowling \& P. Reynolds (Eds.), Making sense of sexual consent (pp. 1-15). Burlington, VT: Ashgate.

Derning, M. E., Krassen Covan, E., Swan, S. C., \& Billings, D. L. (2013). Exploring rape myths, gendered norms, group processing, and the social context of rape among college women. Violence against Women, 19, 465-485. doi: 10.1177/107780121387044 
Dolby, N. (2014). The future of empathy: Teaching the Millennial generation. Journal of College and Character, 15, 39-44. doi: 10.1515/jcc-2015-0006

Donat, P. L. N., \& White, J. W. (2000). Re-examining the issue of nonconsent in acquaintance rape. In C. Brown \& J. W. White (Eds.), Sexuality, society and feminism (Vol. 8, pp. 355376). Washington, DC: American Psychological Association.

Eisenberg, N. (2000). Empathy and sympathy. In M. Lewis \& J. M. Haviland-Jones (Ed.), Handbook of emotions (pp. 677-691). New York, NY: Guilford Press.

Feshbach, N., \& Cohen, S. (1998). Training affects comprehension in younger children: An experimental evaluation. Journal of Applied Developmental Psychology, 9, 201-2010. doi: $10.1016 / 0193-3973(88) 90023-8$

Fisher, B. S., Cullen, F. T., \& Turner, M. G. (2000). The sexual victimization of college women. US Department of Justice. Retrieved December 1, 2016, from https://www.ncjrs.gov/pdffiles1/nij/182369.pdf

Flyntz, L. (2016). Envisioning a culture of consent: A conversation with FORCE. Afterimage, $43(4), 18-22$.

Foubert, J. D., Tatum, J. L., \& Godin, E. E. (2010). First-year male students' perceptions of a rape prevention program 7 months after their participation: Attitude and behavior changes. Journal of College Student Development, 51, 707-715. doi:

$10.1353 / \mathrm{csd} .2010 .0021$

Gagnon, J. H. (1990). The explicit and implicit use of the scripting perspective in sex research. Annual Review of Sex Research, 1, 1-43. doi: 10.1080/10532528.1990.10559854 
Gagnon, J. H., \& Parker, R. G. (1995). Introduction: Conceiving sexuality. In R. G. Parker \& G. H. Gagnon (Eds.), Conceiving sexuality: Approaches to sex research in a postmodern world (pp. 3-16). New York, NY: Routledge.

Galvin, K. M., Braithwaite, D. O., \& Bylund, C. L. (2016). Family communication: Cohesion and change (9 $9^{\text {th }}$ ed.). New York, NY: Routledge.

Garbarino, J., \& Crouter, A. (1978). Defining the community context for parent-child relations: The correlates of child maltreatment. Child Development, 49, 604. doi: 10.2307/1128227

Gray, E. (2014, May 26). Missoula. Time, 183(20), 20-27.

Gowen, L. K., \& Winges-Yanez, N. (2014). Lesbian, gay, bisexual, transgender, queer, and questioning youths' perspectives of inclusive school-based sexuality education. Journal of Sex Research, 51, 788-800. doi: 10.1080/00224499.2013.806648

Guckenheimer, D. (2008). Rape culture. In C. M. Renzetti \& J. L. Edelson (Eds.), Encyclopedia of Interpersonal Violence (pp. 581-582). Thousand Oaks, CA: Sage.

Hall, D. S. (1998, August 10). Consent for sexual behavior in a college student population. Electronic Journal of Human Sexuality, 1. Retrieved from http://www.ejhs.org/volume1/consent1.htm

Hanson, K. A., \& Gidycz, C. A. (1998). An empirical evaluation of a program designed to reduce the risk of multiple sexual victimization. Journal of Interpersonal Violence, 13, 472-488. doi: 1177/088626098013004004

Hatcher, S. L., Nadeau, M. S., Walsh, L. K., Reynolds, M., Galea, J., \& Marz, K. (1994). The teaching of empathy for high school and college students: Testing Rogerian methods with the Interpersonal Reactivity Index. Adolescence, 29, 961-974. 
Heise, L. L. (1998). Violence against women: An integrated, ecological framework. Violence against Women, 4, 262-290. doi: 10.1177/1077801298004003002

Herman, D. (1984). The rape culture. In J. Freeman (Ed.), Women: A feminist perspective. Mountain View, CA: Mayfield.

Hersh, K., \& Gray-Little, B. (1998). Psychopathic traits and attitudes associated with selfreported sexual aggression in college men. Journal of Interpersonal Violence, 13, 456471. doi: $10.1177 / 088626098013004003$

Hickman, S. E., \& Muehlenhard, C. L. (1999). "By the semi-mystical appearance of a condom”: How young women and men communicate sexual consent in heterosexual situations. Journal of Sex Research, 36, 258-272. doi: 10.1080/00224499909551996

Humphreys, T. P. (2004). Understanding sexual consent: An empirical investigation of the normative script for young heterosexual adults. In M. Cowling \& P. Reynolds (Eds.), Making sense of sexual consent (pp. 209-225). Burlington, VT: Ashgate.

Humphreys, T. P. (2007). Perceptions of sexual consent: The impact of relationship history and gender. Journal of Sex Research, 44, 307-315. doi: 10.1080/00224490701586706

Humphreys, T. P., \& Brousseau, M. M. (2010). The Sexual Consent Scale-revised: Development, reliability, and preliminary validity. Journal of Sex Research, 47, 420-428. doi: 10.1080/00224490903151358

Humphreys, T. P., \& Herold, E. (2007). Sexual consent in heterosexual relationships: Development of a new measure. Sex Roles, 57, 305-315. doi: 10.1007/s11199-007-92647 
Hust, S. J. T., \& Brown, J. D. (2008) Gender, media use and effects. In S. L. Calvert \& B. J. Wilson (Eds.), Blackwell handbook for child development and the mass media (pp. 98120). Oxford, UK: Blackwell.

Hust, S. J. T., Lei, M., Ren, C., McNab, A. L., Marett, E. G., \& Willoughby, J. F. (2013). The effects of sports media exposure on college students' rape myth beliefs and intentions to intervene in a sexual assault. Mass Communication and Society, 16, 762-786. doi: $10.1080 / 15205436.2013 .816737$

Hust, S. J. T., Marett, E. G., Ren, C., Adams, P. M., Willoughby, J. F., Lei, M., \& Norman, C. (2014). Establishing and adhering to sexual consent: the association between reading magazines and students' sexual consent negotiation. Journal of Sex Research, 51, 280290. doi: 10.1080.00224499.2012.727914

Johnson, A. M., \& Hoover, S. M. (2015). The potential of sex consent interventions on college campuses: a literature review on the barriers to establishing affirmative sexual consent. PURE Insights, 4(5). Retrieved from http://digitalcommons.wou.edu/pure/vol4/iss1/5

Johnson, T. P. \& van de Vijver, F. J. R. (2002). Social desirability in cross-cultural research. In J. A. Harkness, F. J. R. van d Vijver, \& P. P. Mohler (Eds.), Cross-cultural survey methods (pp. 193-202). Hoboken, NJ: John Wiley.

Jozkowski, K. N. (2011). Measuring internal and external conceptualizations of sexual consent: A mixed-methods exploration of sexual consent (Doctoral dissertation). Retrieved from ProQuest Dissertations and Theses database. (UMI No. 346353)

Jozkowski, K. N., \& Peterson, Z. D. (2013). College students and sexual consent: unique insights. Journal of Sex Research, 50, 517-523. doi: 10.1080/00224499.2012.700739 
Jozkowski, K. N., \& Peterson, Z. D. (2014). Assessing the validity and reliability of the perceptions of the Consent to Sex Scale. Journal of Sex Research, 51, 632-645. doi: 10.1080.00224499.2012.757282

Jozkowski, K. N., Peterson, Z. D., Sanders, S. A., Dennis, B., \& Reece, M. (2014). Gender differences in heterosexual college students' conceptualization and indicators of sexual consent: implications for contemporary sexual assault prevention education. Journal of Sex Research, 51, 904-916. doi: 10.1080/00224499.2013.792326

Jozkowski, K., Sanders, S., Peterson, Z., Dennis, B., \& Reece, M. (2014). Consenting to sexual activity: the development and psychometric assessment of dual measures of consent. Archives of Sexual Behavior, 43, 437-450. doi: 10.1007/s10508-013-0225-7

Kantor, L. M., Santelli, J. S., Teitler, J., \& Balmer, R. (2008). Abstinence only policies and programs: An overview. Sexuality Research and Social Policy, 5(3), 6-17. doi: 10.1525/srsp.2008.5.3.6

Kaufman, M. R., Cornish, F., Zimmerman, R. S., \& Johnson, B. T. (2014). Health behavior change models for HIV prevention and AIDS care. JAIDS Journal of Acquired Immune Deficiency Syndromes, 66, S250-S258. doi: 10.1097/qai.0000000000000236

Keefe, J. W. (2006). Locus of control. In F. W. English (Eds.), Encyclopedia of Educational Leadership and Administration. Thousand Oaks, CA: Sage. doi: $10.4135 / 9781412939584 . n 348$

Knoblach, L. K., Haunani Solomon, D., \& Theiss, J. A. (2006). The role of intimacy in the production and perception of relationship talk within courtship. Communication Research, 33, 211-241. doi: 10.1177/0093650206289148 
Konrath, S. H., O’Brien, E. H., \& Hsing, C. (2011). Changes in dispositional empathy in American college students over time. Personality and Social Psychology Review, 15, 180-198. doi: $10.1177 / 1088868310377395$

Koss, M. P., Dinero, T. E., Seibel, C. A., \& Cox, S. L. (1987). The scope of rape: Incidence and prevalence of sexual aggression and victimization in a national sample of higher education students. Journal of Consulting and Clinical Psychology, 55, 162-170. doi: 10.1037//0022-006x.55.2.162

Krahé, B., Scheinberger-Olwig, R., \& Kolpin, S. (2000). Ambiguous communication of sexual intentions as a risk marker of sexual aggression. Sex Roles, 42, 313-337. doi: 10.1023/A:1007080303569

L'Engle, K. L., Brown, J. D., \& Kenneavy, K. (2006). The mass media are an important context for adolescents' sexual behavior. Journal of Adolescent Health, 38, 186-192. doi: 10.1016/j.jadohealth.2005.03.020

Lake, P. F. (2015). Keynote: The weaponization of alcohol: Effectively linking high-risk alcohol use prevention to campus Title IX efforts [PowerPoint slides]. Retrieved from Higher Education Center for Alcohol and Drug Misuse Prevention and Recovery: http://hecaod.osu.edu/wp-content/uploads/2015/08/Peter-Lake-Keynote_TheWeaponization-of-Alcohol.pdf

Laumann, E. O., \& Gagnon, J. H. (1995). A sociological perspective on sexual action. In R. G. Parker \& J. H. Gagnon (Eds.), Conceiving sexuality: Approaches to sex research in a post modern world. New York, NY: Routledge.

Lim, G. Y., \& Roloff, M. E. (1999). Attributing sexual consent. Journal of Applied Communication Research, 27(1), 1-23. doi: 10.1080/00909889909365521 
Lindgren, K. P., Parkhill, M. R., George, W. H., \& Hendershot, C. S. (2008). Gender differences in perceptions of sexual intent: Qualitative review and integration. Psychology of Women Quarterly, 32, 423-439. doi: 10.111/j.1471-6402.20089.00456.x

Lisak, D., \& Miller, P. M. (2002). Repeat and multiple offending among undetected rapists. Violence and Victims, 17, 73-84. doi: 10.1891/vivi.17.1.73.33638

Long, S. M., Ullman, S. E., Long, L. M., Mason, G. E., \& Starzynski, L. L. (2007). Women's experiences of male perpetrated sexual assault by sexual orientation. Violence and Victims, 22, 684-701. doi: 10.1891/088667007782793138

McGregor, J. (1996). Why when she says no she doesn't mean maybe and doesn't mean yes: A critical reconstruction of consent, sex, and the law. Legal Theory, 2, 175-208. doi: $10.1017 / \mathrm{s} 1352325200000483$

McLeroy, K. R., Bibeau, D., Steckler, A., \& Glanz, K. (1988). An ecological perspective on health promotion programs. Health Education \& Behavior, 15, 351-377. doi: $10.1177 / 109019818801500401$

Masters, T. N., Casey, E., Wells, E. A., \& Morrison, D. M. (2013). Sexual scripts among young heterosexually active men and women: Continuity and change. Journal of Sex Research, 50, 409-420. doi: 10.1080/00224499.2012.661102

Maxwell, Z. (2014, March 27). Rape culture is real. Time. Retrieved from http://time.com/40110/rape-culture-is-real/

Miller-Ott, A. E., \& Linder, A. (2014). Romantic partners' use of facework and humor to communicate about sex. Qualitative Research Reports in Communication, 14, 69-78. doi: $10.1080 / 17459435.2013 .835344$ 
Muehlenhard, C. L., \& Hollabaugh, L. C. (1988). Do women sometimes say no when they mean yes? The prevalence and correlates of women's token resistance to sex. Journal of Personality and Social Psychology, 54, 872-879. doi: 10.1037/022-351.54.5.872

Muehlenhard, C. L., Humphreys, T. P., Jozkowski, K. N., \& Peterson, Z. D. (2016). The complexities of sexual consent among college students: a conceptual and empirical review. Journal of Sex Research, 53, 457-487. doi: 10.1080.00224499.2016.1146651

Muehlenhard, C. L., \& Linton, M. A. (1987). Date rape and sexual aggression in dating situations: Incidence and risk factors. Journal of Counseling Psychology, 34, 186-196. doi: 10.1037//0022-0167.34.2.186

National Association of Student Personnel Administrators. (2004). Leadership of a healthy campus: An ecological approach to student success. Retrieved from: http://docplayer.net/11036490-Leadership-for-a-healthy-campus-an-ecological-approachfor-student-success.html

Olderbak, S., Sassenrath, C., Keller J., \& Wilhelm, O. (2014). An emotion-differentiated perspective on empathy with the emotion specific empathy questionnaire. Frontiers in Psychology, 5, 1-14. doi: 10.3389.fpsyg.2014.00653

Peterson, Z. D., \& Muehlenhard, C. L. (2007). Conceptualizing the "wantedness" of women's consensual and nonconsensual sexual experiences: Implications for how women label their experiences with rape. Journal of Sex Research, 44, 72-88. http://www.jstor.org/stable/20620289

Reherman, T. (1987). Metacommunication strategies for reducing romantic relationship uncertainty. Journal of the Northwest Communication Association, 15, 25-38. 
Reinholtz, R. K., Muehlenhard, C. L., Phelps, J. L., \& Satterfield, A. T. (1995). Sexual discourse and sexual intercourse: How the way we communicate affects the way we think about sexual coercion. In P. J. Kalbfleish \& M. J. Cody (Eds.), Gender, power, and communication in human relationships (pp. 141-162). Hillsdale, NJ: Erlbaum.

Reynolds, P. (2004). The quality of consent: Sexual consent, culture, communication, knowledge and ethics. In M. Cowling \& P. Reynolds (Eds.), Making sense of sexual consent (pp. 93109). Burlington, VT: Ashgate.

Sakaluk, J. K., Todd, L. M., Milhausen, R., Lachowsky, N. J., \& Undergraduate Research Group in Sexuality. (2014). Dominant heterosexual scripts in emerging adulthood: Conceptualization and measurement. Journal of Sex Research, 51, 516-531. doi: 10.1080.00224499.2012.745473

Satir, V. (1967). Conjoint family therapy (Rev. Ed.). Palo Alto, CA: Science \& Behavior Books. Skinner, E., \& Greene, T. (2008). Perceived control, coping, and engagement. In T. L. Good (Ed.), 21 st Century education handbook (pp. 121-130). Thousand Oaks, CA: Sage.

So, J., Achar, C., Han, D., Agrawal, N., Duhachek, A., \& Maheswaran, D. (2015). Psychology of appraisal emotions and decision making. Journal of Consumer Psychology, 25, 359-371. doi: $10.1016 /$ j.jcps.2015.04.003

Spence, J. T., Losoff, M., \& Robbins, A. S. (1991). Sexually aggressive tactics in dating relationships: Personality and attitudinal correlates. Journal of Social and Clinical Psychology, 10, 289-304. doi: 10.1521/jscp.1991.10.3.289 
Sprecher, S., Hatfield, E., Cortese, A., Potapova, E., \& Levitskaya, A. (1994). Token resistance to sexual intercourse and consent to unwanted sexual intercourse: College students’ dating experiences in three countries. Journal of Sex Research, 31, 125-132. doi: 10.1080 .00224499409551739

Spreng, R. N., McKinnon, M. C., Mar, R. A., \& Levine, B. (2009). The Toronto Empathy Questionnaire: Scale development and initial validation of a factor-analytic solution to multiple empathy measures. Journal of Personality Assessment, 91, 62-71. doi: $10.1080 / 00223890802484381$

Steele, C. M., \& Josephs, R. A. (1990). Alcohol myopia: Its prized and dangerous effects. American Psychologist, 45, 921-933. doi: 10.1037/0003-0066X.45.8.921

Struckman-Johnson, C. (1988). Forced sex on dates: It happens to men too. The Journal of Sex Research, 24, 234-241. doi: 10.1080/00224498809551418

Task Force of the National Advisory Council on Alcohol Abuse and Alcoholism. (2002). Highrisk drinking in college: What we know and what we need to know. Retrieved from https://www.collegedrinkingprevention.gov/media/finalpanel1.pdf

Thornton, S., Todd, B., \& Thornton, D. (1996). Empathy and the recognition of abuse. Legal and Criminological Psychology, 1, 147-153.

Tolan, P. H., \& Guerra, N. (1994). Prevention of delinquency: Current status and issues. Applied and Preventive Psychology, 3, 251-273. doi: 10.1016/s0962-1849(05)80098-8

Turkle, S. (2011). Alone together: Why we expect more from technology and less from each other. New York, NY: Basic Books. 
Vachon, D. D., Lynam, D. R., \& Johnson, J. A. (2014). The (non)relation between empathy and aggression: Surprising results from a meta-analysis. Psychological Bulletin, 140, 751773. doi: $10.1037 / \mathrm{a} 0035236$

Vandiver, D. M., \& Rager Dupalo, J. (2012). Factors that affect college students' perceptions of rape: What is the role of gender and other situational factors? International Journal of Offender Therapy and Comparative Criminology, 57, 592-612. doi: $1001177 / 0306624 X 12436797$

Visschers, J., Jaspaert, E., \& Vervaeke, G. (2017). Social desirability in intimate partner violence and relationship satisfaction report: An exploratory analysis. Journal of Interpersonal Violence, 32, 1401-1420. doi: 10.1177/0886260515588922

Wallace, S. A., Miller, K. S., \& Forehand, R. (2008). Perceived peer norms and sexual intentions among African American preadolescents. AIDS Education \& Prevention, 20, 360-369. doi: 10.1521/aeap.2008.20.4.360

Ward, R. M., Matthews, M. R., Weiner, J., Hogan, K. M., \& Popson, H. C. (2012). Alcohol and sexual consent scale: Development and validation. American Journal of Health Behavior, 36, 746-756. doi: 10.5993/AJHB.36.6.3

Watzlawick, P., Beavin, J. H., \& Jackson, D. D. (1967). Pragmatics of human communication: A study of interactional patterns, pathologies, and paradoxes. New York, NY: Norton.

Werder, O. H. (2009). Media effects theories. In S. W. Littlejohn \& K. A. Foss (Eds.). Encyclopedia of communication theory. Thousand Oaks, CA: Sage.

West, R. (1996). A comment on consent, sex, and rape. Legal Theory, 2, 223-251. doi: $10.1017 / \mathrm{s} 1352325200000501$ 
White House Council on Women and Girls. (2014). Rape and sexual assault: A renewed call to action. Retrieved from https://www.whitehouse.gov/sites/default/files/docs/sexual_assault_report_1-21-14.pdf 


\section{APPENDIX: SURVEY INSTRUMENT}

Directions: Your participation in this study will help us learn more about college students' communication, behaviors, and opinions about sexual consent. To being this survey, we need to learn a little more about you. Please know that all survey responses will be confidential and that researchers will only report the average of all respondents' answers.

\section{Demographics (5 questions)}

1. What is your age? <number slider>

2. What is your year in School:

First year

Second year

Third year

Fourth year

Graduate student

Other (please specify):

3. What is the gender that you identify as?

Agender

Female

Fluid / Genderqueer

Male

Questioning / Unsure

Other:

4. What is your sexual orientation?

Asexual

Bisexual

Gay/Lesbian

Heterosexual (straight)

Pansexual

Questioning / Unsure

Other:

5. Please select which of the following you most identify with:

African American / Black

Asian

Caucasian / White

Hispanic / Latino(a)

Multiracial

Native American

Pacific Islander

I do not wish to disclose

Other (please specify): 


\section{Currently Sexual Activity (3 questions)}

6. Please select your current sexual activity status:

Committed relationship with one sexual partner

One, non-committed sexual partner

Multiple, non-committed sexual partners

Not currently sexually active, but have been in the past

Other, please specify:

7. If you selected committed relationship with one sexual partner, please indicate how many months you have been in your current relationship:

$<$ number slider $>$

8. How often do you engage in sexual activity when under the influence of alcohol?
Never
Rarely
Sometimes
Most of the Time
Always

\section{Sex Consent \& Sexual Preparedness Questions (5 questions)}

9. Has anyone directly discussed sexual consent with you?

Yes

No

Not sure

10. If you selected yes above, please select who has spoken with you about sexual consent. Please select all that apply.

Friend

Parent/Guardian

School / Sex Education

Sibling

Not applicable

Other, please specify

11. Please rate the following individuals' influence on your perceptions of sexual consent with one being most influential and 7 being least influential. [R]

Friend(s)

Media (i.e. internet, magazines, movies, television, etc.)

Parent(s)/Guardian(s)

Religion

School

Sibling(s)

Other

Directions: Please respond to the following two questions giving yourself a percentage, like a grade with 0 meaning you are not adequately prepared and 100\% meaning you are perfectly prepared.

12. I feel that I am adequately prepared to navigate sexual situations. $\%$ 
13. I feel that I am adequately prepared to talk about consent with my sexual partner(s). $\%$

NOTE: All of the following scales are 5-point Likert. R denotes options that were reverse coded during analysis.

Directions: This section will help us better understand how you interact with others. Please answer each question with a response between 1 and 5, with 1 meaning strongly disagree and 5 meaning strongly agree.

\section{Scale 1: Empathy (16 questions)}

14. When someone else is feeling excited, I tend to get excited too

15. Other people's misfortunes do not disturb me a great deal [R]

16. It upsets me to see someone being treated disrespectfully

17. I remain unaffected when someone close to me is happy [R]

18. I enjoy making people feel better

19. I have tender, concerned feelings for people less fortunate than me

20. When a friend starts talking about his/her problems, I try to steer the conversation towards something else $[\mathrm{R}]$

21. I can tell when others are sad even when they do not say anything

22. I find that I am "in tune" with other people's moods

23. I do not feel sympathy for people who cause their own serious illnesses [R]

24. I become irritated when someone cries [R]

25. I am not really interested in how other people feel [R]

26. I get a strong urge to help when I see someone who is upset

27. When I see someone being treated unfairly, I do not feel very much pity for them [R]

28. I find it silly for people to cry out of happiness [R]

29. When I see someone being taken advantage of, I feel kind of protective towards them

\section{Scale 2: Perceived Behavioral Control (11 questions)}

Directions: This section will help us learn more about how prepared you feel to obtain consent in a variety of situations. Please answer each question with a response between 1 and 5, with 1 meaning strongly agree and 5 meaning strongly disagree.

30. I would have difficulty asking for consent because it would spoil the mood

31. I am worried that my partner might think I'm weird or strange if I asked for sexual consent before starting any sexual activity

32. I would have difficulty asking for consent because it doesn't really fit with how I like to engage in sexual activity

33. I have not asked for sexual consent at times because I felt that it might backfire and I wouldn't end up having sex

34. I think verbally asking for consent is awkward

35. I have not given my consent for sexual activity at times because I felt that it might backfire and I wouldn't end up having sex

36. I believe that verbally asking for sexual consent reduces the pleasure of the encounter 
37. I would have a hard time verbalizing my consent in a sexual encounter because I am too shy

38. I feel confident that I could ask for sexual consent from a new sexual partner [R]

39. I would not want to ask a partner for consent because it would remind me that I'm sexually active

40. I feel confident that I could ask for consent from my current partner [R]

\section{Scale 3: Attitude toward Establishing Consent (11 questions)}

Directions: This section explores how you feel about establishing consent. Please answer each question with a response between 1 and 5, with 1 meaning strongly disagree and 5 meaning strongly agree.

41. I feel that sexual consent should always be obtained before the start of any sexual activity

42. I believe that asking for sexual consent is in my best interest because it reduces any misinterpretations that might arise

43. I think it is equally important to obtain sexual consent in all relationships regardless of whether or not they have had sex before

44. I feel that verbally asking for sexual consent should occur before proceeding with any sexual activity

45. When initiating sexual activity, I believe that one should always assume they do not have sexual consent

46. I believe that it is just as necessary to obtain consent for genital fondling as it is for sexual intercourse

47. Most people that I care about feel that asking for sexual consent is something I should do

48. I think that consent should be asked before any kind of sexual behavior, including kissing or petting

49. I feel it is the responsibility of both partners to make sure sexual consent is established before sexual activity begins

50. Before making sexual advances, I think that one should assume "no" until there is clear indication to proceed

51. Not asking for sexual consent some of the time is okay $[R]$

\section{Scale 4: Approaches to Establishing Consent (6 questions)}

Directions: This section will explore how you establish consent. Please answer each question with a response between 1 and 5, with 1 meaning strongly disagree and 5 meaning strongly agree.

52. Typically I communicate sexual consent to my partner using nonverbal signals and body language

53. It is easy to accurately read my current (or most recent) partner's nonverbal signals as indicating consent or non-consent to sexual activity

54. Typically I ask for consent by making a sexual advance and waiting for a reaction, so I know whether or not to continue 
55. I don't have to ask or give my partner sexual consent because my partner knows me well enough

56. I don't have to ask or give my partner sexual consent because I have a lot of trust in my partner to "do the right thing",

57. I always verbally ask for consent before I initiate a sexual encounter [R]

\section{Scale 5: Consent Norms (8 questions)}

Directions: This section will help us better understand your overall opinions about sexual consent. Please answer each question with a response between 1 and 5, with 1 meaning strongly disagree and 5 meaning strongly agree.

58. I think that obtaining sexual consent is more necessary in a new relationship than in a committed relationship

59. I think that obtaining sexual consent is more necessary in a casual sexual encounter than in a committed relationship

60. I believe that the need for asking for sexual consent decreases as the length of an intimate relationship increases

61. I believe it is enough to ask for consent at the beginning of a sexual encounter

62. I believe that sexual intercourse is the only sexual activity that requires explicit verbal consent

63. I believe that partners are less likely to ask for sexual consent the longer they are in a relationship

64. If consent for sexual intercourse is established, petting and fondling can be assumed

65. I have not given much thought to the topic of sexual consent $[R]$

\section{Scale 6: Ongoing Negotiation (8 questions)}

Directions: This section explores how you negotiate consent during sexual situations. Please answer each question with a response between 1 and 5, with 1 meaning strongly disagree and 5 meaning strongly agree.

66. During a sexual encounter, I typically only GIVE my consent once [R]

67. During a sexual encounter, I typically only ASK for consent once [R]

68. During a sexual encounter, I typically GIVE consent for each new sexual activity

69. During a sexual encounter, I typically ASK for consent for each new sexual activity

70. During a sexual encounter, I check in with my partner periodically to make sure they are still interested in continuing the encounter

71. If a sexual request is made and the partner indicates "no," it is okay to continue negotiating the request $[\mathrm{R}]$

72. I tend to NOT decide ahead of time what I will and will not consent to sexually and wait until I am "in the moment" to decide

73. I feel comfortable withdrawing my consent at any time and ending a sexual encounter 


\section{Scale 7: Metacommunication (5 questions)}

Directions: This section asks questions about discussions you have had about sexual consent. Please answer each question with a response between 1 and 5, with 1 meaning strongly disagree and 5 meaning strongly agree.

74. I have discussed engaging in sexual activity with my partner(s) at times other than during sexual encounters

75. I have discussed sexual consent with my partner(s) at times other than sexual encounters

76. Too few sexual partners openly discuss sexual consent

77. I have discussed sexual consent with a friend

78. I have heard sexual consent being discussed by other students on campus 